\title{
THE GROMOV NORM OF THE KAEHLER CLASS AND THE MASLOV INDEX*
}

\author{
JEAN-LOUIS CLERC ${ }^{\dagger}$ AND BENT ØRSTED $\ddagger$
}

\begin{abstract}
Let $\mathcal{D}$ be a Hermitian symmetric space of the non-compact type, $\omega$ its Kaehler form. For $\Delta$ a geodesic triangle in $\mathcal{D}$, we compute explicitly the integral $\int_{\Delta} \omega$, generalizing previous results (see [D-T]). As a consequence, if $X$ is a manifold which admits $\mathcal{D}$ as universal cover, we calculate the Gromov norm of $[\omega] \in H^{2}(X, \mathbb{R})$. The formula for $\int_{\Delta} \omega$ is extended to ideal triangles. Precise estimates are given and triangles for which the bound is achieved are studied. For tube-type domains we show the connection of these integrals with the Maslov index we introduced in a previous paper (see [C-Ø]).
\end{abstract}

0. Introduction. Let $M$ be a Hermitian symmetric space of the non-compact type, which for simplicity, we assume to be irreducible. Let $G$ be the neutral component of the group of biholomorphic automorphisms of $M$. The space $M$ admits a natural ( $G$-invariant) Kaehler form $\omega$. This real differential form of degree 2 is closed and hence can be integrated along any 2-cycle, in particular geodesic triangles (to mean triangles the sides of which are geodesic segments). When $M$ is of type I,II or III (in E. Cartan's classification), the integral $\int_{\Delta} \omega$ (the symplectic area of the geodesic triangle $\Delta$ ) was computed in [D-T]. By using their techniques, we give the result in the general case. It turns out that these quantities have an upper bound, and with the appropriate normalization, the bound depends only on the rank $r$ of $M$. We extend these computations to ideal triangles, and we prove (new) sharp estimates for the areas. In particular, we determine precisely the triangles for which the upper bound is achieved. This turns out be of great geometric significance, as the summits of such an extremal triangle are contained in the image of a tight holomorphic totally geodesic imbedding of the complex unit disc into $M$ (Theorem 4.7). Generally speaking, our study of the integrals $\int_{\Delta} \omega$ requires the fine structure of Hermitian symmetric spaces : special rôle played by the tube-type case, behaviour of geodesics at infinity and structure of $G$-orbits in the boundary, use of partial Cayley transforms.

This study is also related to a previous work (see [C- $\varnothing]$ ) where we extended the notion of Maslov index to the Shilov boundary $S$ of a Hermitian symmetric space of tube-type. The Maslov index is (up to a factor $\pi$ ) nothing but the symplectic area of ideal triangles with summits in $S$, and in some sense the present work can be understood as a continuation of $[\mathrm{C}-\varnothing]$.

The computation of the integrals was used in [D-T] to calculate the Gromov norm of the Kaehler class of a compact Hermitian locally symmetric manifold $X=\Gamma \backslash M$, where $M$ is of type I and $\Gamma$ a discrete, torsion-free, co-compact subgroup of the group $G$. They observed that it has a nice topological corollary. Let $S$ be a Riemann surface of genus $g>1$ and $f: S \rightarrow X$ a continuous map. Then

$$
\left|\int_{S} f^{*} \omega\right| \leq 4 r(g-1) \pi
$$

\footnotetext{
*Received February 18, 2003; accepted for publication June 17, 2003.

†Institut Elie Cartan, U.M.R. 7502, Université Henri Poincaré B.P. 239, 54506 Vandœuvre-lèsNancy Cedex, France (clerc@iecn.u-nancy.fr).

†Institut for Matematik og Datalogi, Odense Universitet, DK-5230 Odense M Danemark (orsted@imada.ou.dk).
} 
These results are valid for the general case.

Let us mention other connections of this work which could yield further developments. First, the integral $\int_{\Delta} \omega$ gives a concrete realization of a bounded 2-cohomology class of $G$. The space $H_{b}^{2}(G)$ (and more generally bounded cohomology) has been intensively studied by several authors (see references in $[M]$ ). Second, the new information on this cocycle could help to investigate rigidity problems in Kaehler geometry (in the spirit of [T2]) and also the space of representations of the fundamental group of a surface of genus $g>1$ in $G$ (see [Got] for a most recent reference). More specifically, our results could be used to study the maximal representations of surface groups in bounded symmetric domains, generalizing Hernandez's work (see $[\mathrm{H}]$, and recently [B-G-G]). In fact M. Burger, A. Iozzi and A. Wienhard informed us that they obtain new results (cf $[B-I-W]$ ) in this direction by combining their own techniques (see e.g. [B-I]) with some of the results of the present paper.

Section 1 introduces the geometry of the Hermitian symmetric spaces, with a few technical results (e.g. on the (normalized) Bergman kernel) which will be needed in section 3 and 4 . Section 2 is the computation of the integrals $\int_{\Delta} \omega$ where we follow $[D-T]$. Section 3 gives the corresponding estimate of these integrals, and our method of proof is different from that in [D-T]. In section 4 we introduce the ideal triangles, extend the definition, establish sharp estimates and discuss the extrema of $\int_{\Delta} \omega$ for these triangles. These results are new even in the classical cases. This is the most technical part, as it requires to realize the Hermitian space $M$ as a Siegel domain of type III. Section 5 follows [D-T] closely to give some topological consequences of the estimates.

The authors would like to thank J. Faraut, who, after reading [C- $\varnothing]$, indicated the reference [D-T] which was crucial for the development of the present work, and Anna Wienhard, whose challenging questions contributed to a reformulation of Theorem 4.7 .

1. Geometric setting. Our main references for this section are $[\mathrm{S}]$ and part III of [F-al]. Let $M$ be an irreducible Hermitian symmetric space of the non-compact type. Let $G$ be the neutral component of the group of biholomorphic mappings of $M$. Fix a base point $o \in M$, and let $K$ be the stabilizer of $o$ in $G$. Then $K$ is a maximal compact subgroup of $G$. Denote by $\theta$ the associated Cartan involution of $G$, and let

$$
\mathfrak{g}=\mathfrak{k} \oplus \mathfrak{p}
$$

be the corresponding Cartan decomposition of $\mathfrak{g}$. Let $J$ be the complex structure on $\mathfrak{p}$, and let $H_{0}$ be the (unique) element in the center of $\mathfrak{k}$ such that ad $H_{0 \mid \mathfrak{p}}=J$. The complexification $\mathfrak{p}_{\mathbb{C}}$ splits as $\mathfrak{p}_{\mathbb{C}}=\mathfrak{p}_{+} \oplus \mathfrak{p}_{-}$(eigenspace decomposition with respect to $J)$, and let

$$
\mathfrak{g}_{\mathbb{C}}=\mathfrak{p}_{+} \oplus \mathfrak{k}_{\mathbb{C}} \oplus \mathfrak{p}_{-}
$$

the corresponding grading of $\mathfrak{g}_{\mathbb{C}}$. Let $G_{\mathbb{C}}$ be a connected Lie group with Lie algebra $\mathfrak{g}_{\mathbb{C}}$ and let $P_{+}, K_{\mathbb{C}}, P_{-}$be the analytic subgroups of $G_{\mathbb{C}}$ corresponding to $\mathfrak{p}_{+}, \mathfrak{k}_{\mathbb{C}}, \mathfrak{p}_{-}$. The map

$$
\left(p_{+}, k, p_{-}\right) \longmapsto p_{+} k p_{-} \quad P_{+} \times K_{\mathbb{C}} \times P_{-} \longrightarrow G_{\mathbb{C}}
$$

is injective with dense open image, and we denote the corresponding decomposition of an element $g \in G$ by

$$
g=(g)_{+}(g)_{0}(g)_{-} .
$$


For $g \in G_{\mathbb{C}}$ and $z \in \mathfrak{p}_{+}$such that $g \exp (z) \in P_{+} K_{\mathbb{C}} P_{-}$, define $g(z) \in \mathfrak{p}^{+}$and $J(g, z) \in$ $K_{\mathbb{C}}$ by

$$
\exp g(z)=(g \exp z)_{+}, \quad J(g, z)=(g \exp z)_{0} .
$$

The expression $J(g, z)$ is the canonical automorphy factor (see [S]).

The automorphy factor satisfies a cocycle formula :

$$
J\left(g g^{\prime}, z\right)=J\left(g, g^{\prime}(z)\right) J\left(g^{\prime}, z\right)
$$

Denote by $\sigma$ the conjugation of $G_{\mathbb{C}}$ with respect to $G$. Observe that

$$
\sigma\left(P_{ \pm}\right) \subset P_{\mp}, \quad \sigma\left(K_{\mathbb{C}}\right) \subset K_{\mathbb{C}}
$$

For $z, w \in \mathfrak{p}_{+}$such that $\exp (-\sigma(w)) \exp z \in P_{+} K_{\mathbb{C}} P_{-}$define $K(z, w)$ by

$$
K(z, w)=J(\exp (-\sigma(w)), z)^{-1}=\left((\exp (-\sigma(w)) \exp z)_{0}\right)^{-1} .
$$

The automorphy kernel $K(z, w)$ satisfies the following relations :

$$
\begin{gathered}
K(w, z)=\sigma(K(z, w))^{-1} \\
K(g(z), \sigma(g)(w))=J(g, z) K(z, w) \sigma(J(\sigma(g), w))^{-1}
\end{gathered}
$$

for $z, w \in \mathfrak{p}_{+}, g \in G_{\mathbb{C}}$, whenever the expressions are defined.

The space $M$ can be realized as a bounded domain $\mathcal{D}$ in $\mathfrak{p}_{+}$by the Harish Chandra imbedding

$$
g K \longmapsto \exp ^{-1}\left(g_{+}\right), \quad G / K \longrightarrow \mathfrak{p}_{+} .
$$

When $z \in \mathcal{D}$ and $g \in G$, the automorphy factor $J(g, z)$ is always defined, and similarly, if $z, w \in \mathcal{D}$, then the automorphy kernel $K(z, w)$ is defined.

For $z \in \mathcal{D}, K(z, z)$ is well-defined and satisfies $\sigma(K(z, z))=K(z, z)^{-1}$. As the restriction of $\sigma$ to $K_{\mathbb{C}}$ is a Cartan involution, this shows that $K(z, z)$ belongs to exp $i \mathfrak{k}$ so that there is a well-defined square $\operatorname{root} K(z, z)^{\frac{1}{2}}$ in $K_{\mathbb{C}}$. Set

$$
g_{z}=\exp (z) K(z, z)^{\frac{1}{2}} \exp (\sigma(z)) .
$$

The element $g_{z}$ belongs to $G$ and satisfies $g_{z}(0)=z$. It is in fact the only element $g \in \exp (\mathfrak{p})$ such that $g(0)=z$ (see [S] p. 71).

On $\mathfrak{p}_{+}$there is a standard Hermitian inner product associated to the Killing form $B$ of $\mathfrak{g}$. There corresponds a Lebesgue measure on $\mathfrak{p}_{+}$and a Bergman space for $\mathcal{D}$. The corresponding Bergman kernel is given by

$$
k_{\mathcal{D}}(z, w)=c\left(\operatorname{det} \operatorname{Ad}_{\mid \mathfrak{p}_{+}} K(z, w)\right)^{-1}
$$

where $c$ is a positive real number which will play no role in this paper. In fact we ignore this and set $c=1$, so that in particular $k_{\mathcal{D}}(z, z)=1$ for $z \in \mathcal{D}$. Its transformation law under the action of $G$ is given by

$$
k_{\mathcal{D}}(g z, g w)=j(g, z)^{-1} k_{\mathcal{D}}(z, w) \overline{j(g, w)}^{-1}
$$


where $j(g, z)=\operatorname{det} \operatorname{Ad}_{\mid \mathfrak{p}_{+}} J(g, z)$. To the Bergman kernel is associated the Bergman metric, which turns $\mathcal{D}$ into a complex Hermitian symmetric space.

Let $\mathfrak{h}$ be a maximal abelian subalgebra of $\mathfrak{k}$, and let $\Delta\left(\mathfrak{g}_{\mathbb{C}}, \mathfrak{h}_{\mathbb{C}}\right)$ be the corresponding set of roots. As $H_{0}$ belongs to $\mathfrak{h}$, the space $\mathfrak{p}_{+}$is stable by ad $\mathfrak{h}$. The roots $\gamma \in \Delta\left(\mathfrak{g}_{\mathbb{C}}, \mathfrak{h}_{\mathbb{C}}\right)$ such that $\mathfrak{g}_{\gamma} \subset \mathfrak{p}_{+}$are said to be positive non-compact, and we denote by $\Phi$ the set of such roots. Let $\gamma \in \Phi$. Then on may choose elements $\widetilde{H}_{\gamma} \in \mathfrak{h}, E_{\gamma} \in \mathfrak{g}_{\gamma}, E_{-\gamma} \in \mathfrak{g}_{-\gamma}$, such that

$$
\gamma(H)=2 \frac{B\left(H, \widetilde{H}_{\gamma}\right)}{B\left(\widetilde{H}_{\gamma}, \widetilde{H}_{\gamma}\right)}, \quad\left[E_{\gamma}, E_{-\gamma}\right]=\widetilde{H}_{\gamma}, \quad \sigma\left(E_{\gamma}\right)=E_{-\gamma}
$$

Let

$$
H_{\gamma}=i \widetilde{H}_{\gamma}, \quad X_{\gamma}=E_{\gamma}+E_{-\gamma}, \quad Y_{\gamma}=-i\left(E_{\gamma}-E_{-\gamma}\right) .
$$

The element $H_{\gamma}$ belongs to $\mathfrak{h}$, and the elements $X_{\gamma}$ and $Y_{\gamma}$ belong to $\mathfrak{p}$. They satisfy the relations

$$
\left[H_{\gamma}, X_{\gamma}\right]=-2 Y_{\gamma}, \quad\left[H, Y_{\gamma}\right]=2 X_{\gamma}, \quad\left[X_{\gamma}, Y_{\gamma}\right]=2 H_{\gamma}
$$

Lemma 1.1. There exists a set $\Gamma=\left\{\gamma_{1}, \ldots, \gamma_{r}\right\}$ in $\Phi$ such that

$$
\mathfrak{a}=\sum_{j=1}^{r} \mathbb{R} X_{\gamma_{j}}
$$

is a Cartan subspace of the pair $(\mathfrak{g}, \mathfrak{k})$.

This is the classical Harish Chandra construction by induction, using a maximal set of strongly orthogonal roots in $\Phi$. For simplicity, let us set for $1 \leq j \leq r$

$$
H_{j}=H_{\gamma_{j}}, E_{j}=E_{\gamma_{j}}, X_{j}=X_{\gamma_{j}}, Y_{j}=Y_{\gamma_{j}}
$$

Let $\mathfrak{a}^{*}$ denote the dual of $\mathfrak{a}$ and let $\left\{\xi_{1}, \xi_{2}, \ldots, \xi_{r}\right\}$ be the basis of $\mathfrak{a}^{*}$ dual to $\left\{X_{1}, X_{2}, \ldots, X_{r}\right\}$. The restricted root system of $\mathfrak{g}$ relative to $\mathfrak{a}$ is of type $C_{r}$ or $B C_{r}$ given by

$$
\begin{gathered}
\pm 2 \xi_{j} \text { each with multiplicity } 1 \\
\pm \xi_{j} \pm \xi_{k}(j \neq k) \text { each with multiplicity } a
\end{gathered}
$$

and possibly

$$
\pm \xi_{j} \text { with multiplicity } b
$$

Let

$$
\mathfrak{a}_{+}=\bigoplus_{j=1}^{r} \mathbb{R} E_{j} \subset \mathfrak{p}_{+}
$$

As $X \longmapsto \frac{1}{2}(X-i J X)$ is a (real) $K$-isomorphism from $\mathfrak{p}$ onto $\mathfrak{p}_{+}$, any element of $\mathfrak{p}_{+}$is conjugate under $K$ to an element of $\mathfrak{a}_{+}$. Hence a $K$-invariant polynomial on 
$\mathfrak{p}_{+}$is determined by its restriction to $\mathfrak{a}_{+}$. Denote by $h(z)$ the (unique) $K$-invariant polynomial on $\mathfrak{p}_{+}$such that

$$
h\left(\sum_{j=1}^{r} t_{j} E_{j}\right)=\prod_{j=1}^{r}\left(1-t_{j}^{2}\right) .
$$

The existence of such a polynomial is a consequence of Chevalley's theorem. As $h$ is real-valued, we may polarize it to get a polynomial on $\mathfrak{p}_{+} \times \mathfrak{p}_{+}$, denoted by $h(z, w)$, holomorphic in $z$ and antiholomorphic in $w$ such that $h(z, z)=h(z)$. In terms of this polynomial, the Bergman kernel for $\mathcal{D}$ is given by the following formula

$$
k_{\mathcal{D}}(z, w)=h(z, w)^{-p}
$$

where $p=(r-1) a+b+2$ (see [F-al] III, prop. V.3.7).

For further purpose, it is better to use a different normalisation for the Hermitian metric, so that the sectional holomorphic curvature has minimal value -1 . Let us compute this minimal value for the Bergman metric.

From the choices made for $H_{\alpha}, E_{\alpha}, E_{-\alpha}, X_{\alpha}, Y_{\alpha}$, we get easily that

$$
B\left(E_{\alpha}, E_{-\alpha}\right)=\frac{1}{2} B\left(\widetilde{H}_{\alpha}, \widetilde{H}_{\alpha}\right), B\left(X_{\alpha}, X_{\alpha}\right)=B\left(Y_{\alpha}, Y_{\alpha}\right)=B\left(\widetilde{H}_{\alpha}, \widetilde{H}_{\alpha}\right)
$$

for any non-compact positive root $\alpha$. For $\alpha=\gamma_{j}$, let us compute $B\left(X_{j}, X_{j}\right)$. As $X_{j}$ belongs to $\mathfrak{p}$,

$$
\left.B\left(X_{j}, X_{j}\right)=2 \operatorname{tr}\left(\left(\operatorname{ad} X_{j}\right)^{2}\right)_{\mid \mathfrak{p}}\right) .
$$

As $\left(\operatorname{ad} X_{j}\right)^{2}=\left(\operatorname{ad} E_{j}+\operatorname{ad} E_{-j}\right)^{2}$, it is useful to use the complexification $\mathfrak{p}_{\mathbb{C}}=\mathfrak{p}_{+} \oplus \mathfrak{p}_{-}$ to compute this trace. The contribution to the trace is obtained from two terms corresponding to $\mathfrak{p}_{+}$and $\mathfrak{p}_{-}$. On $\mathfrak{p}_{+}$, the contribution is merely given by the trace of ad $E_{j} \circ$ ad $E_{-j}$ restricted to $\mathfrak{p}_{+}$. But this operator coincides on $\mathfrak{p}_{+}$with the operator $\operatorname{ad} E_{j} \circ \operatorname{ad} E_{-j}-\operatorname{ad} E_{-j} \circ \operatorname{ad} E_{j}=\operatorname{ad}\left[E_{j}, E_{-j}\right]=\operatorname{ad} \widetilde{H}_{j}$. The computation of $\operatorname{tr} \operatorname{ad} \widetilde{H}_{j}$ is easy from the knowledge of the system of restricted roots so that

$$
\operatorname{tr} \operatorname{ad} \widetilde{H}_{j_{\mid \mathfrak{p}_{+}}}=p .
$$

The computation for $\mathfrak{p}_{-}$is similar, so that eventually $B\left(X_{j}, X_{j}\right)=4 p$ for any $j, 1 \leq$ $j \leq r$.

We identify the tangent space at $o$ with $\mathfrak{p}$. Then, the quadratic form corresponding to the Bergman metric on $\mathfrak{p}$ is $q_{0}=\frac{1}{2} B_{\mid \mathfrak{p}}$ (see $[\mathrm{S}] \mathrm{p}$. 74). The corresponding curvature tensor is

$$
R(X, Y, Z, T)=\frac{1}{2} B([[X, Y], Z], T)
$$

and the holomorphic sectional curvature at $o$ is given by

$$
H(U)=\frac{1}{2} B([[U, J U], U], J U)=\frac{1}{2} B([U, J U],[U, J U])
$$

for $U$ a vector of length 1 in $\mathfrak{p}$. Because of invariance of the curvature, it is sufficient to calculate the holomorphic sectional curvature on an element in the Cartan subspace a. So let $U=\sum_{i=1}^{r} t_{j} X_{j}$. As the $X_{j}$ are mutually orthogonal, we get

$$
q_{0}(U)=\sum_{j=1}^{r} t_{j}^{2} q_{0}\left(X_{j}\right)=2 p \sum_{j=1}^{r} t_{j}^{2}
$$


Note that $J X_{j}=Y_{j},\left[X_{j}, J X_{j}\right]=\left[X_{j}, Y_{j}\right]=-2 i H_{j}$. Hence, from this and the strong orthogonality of the roots $\gamma_{j}$, we get

$$
[U, J U]=\left[\sum_{j=1}^{r} t_{j} X_{j}, \sum_{j=1}^{r} t_{j} Y_{j}\right]=-2 i \sum_{j=1}^{r} t_{j}^{2} H_{j}
$$

so that

$$
H(U)=-2 B\left(\sum_{j=1}^{r} t_{j}^{2} H_{j}, \sum_{j=1}^{r} t_{j}^{2} H_{j}\right)=-2 \sum_{j=1}^{r} t_{j}^{4} B\left(H_{j}, H_{j}\right)=-8 p \sum_{j=1}^{r} t_{j}^{4} .
$$

Under the constraint $q_{0}(U)=1$, the holomorphic sectional curvature reaches its minimal value for all $t_{j}$ equal to 0 except (say) $t_{1}=(2 p)^{-\frac{1}{2}}$, so that the minimal value of the curvature is $-\frac{2}{p}$. Its maximal value is obtained for all $t_{j}$ 's equal and an easy computation shows that it is $-\frac{2}{r p}$.

If one multiplies the metric by a factor $c>0$, then the holomorphic sectional curvature is multiplied by $\frac{1}{c}$. So the proper normalization for the metric is to use $\frac{2}{p}$ times the Bergman metric. It amounts to use the normalized Bergman kernel

$$
k(z, w)=k_{\mathcal{D}}(z, w)^{\frac{2}{p}}=h(z, w)^{-2}
$$

instead of $k_{\mathcal{D}}(z, w)$.

The corresponding transformation law under $G$ is

$$
k(g z, g w)=j(g, z)^{-\frac{2}{p}} k(z, w) \overline{j(g, w)}^{-\frac{2}{p}} .
$$

Observe that for a given $g \in G$ the expression " $j(g, z)^{-\frac{2}{p}}$ " can be defined as $\mathcal{D}$ is simply connected, and the choice depends on a factor of modulus 1 , so that there is no ambiguity in (1.3).

There is a further property of the kernel $k$ which will be needed. This is a subtle relation with the corresponding kernel $k^{T}$ for a subddomain of tube type $\mathcal{D}^{T}$ of $\mathcal{D}$ to be defined below.

Let $Y_{\Gamma}=\sum_{j=1}^{r} Y_{j}$ and define the Cayley transform

$$
c=c_{\Gamma}=\exp \frac{\pi}{4} i Y_{\Gamma}
$$

Then $\operatorname{Ad}\left(c^{4}\right)$ is an involution of $\mathfrak{g}_{\mathbb{C}}$ which preserves $\mathfrak{g}$ and commutes with $\theta$. Denote by $\mathfrak{g}^{T}$ its fixed points. Then $\mathfrak{g}^{T}=\mathfrak{k}^{T} \oplus \mathfrak{p}^{T}$ is a Cartan decomposition, and the corresponding symmetric space is still Hermitian, and now of tube-type. As a bounded domain, it can be realized as $\mathcal{D}^{T}=\mathcal{D} \cap \mathfrak{p}_{+}^{T}$.

The relation to be explained is a special case of a more general result for $K$ invariant polynomials on $\mathfrak{p}$. Denote by $\mathcal{P}$ the space of real-valued polynomials on $\mathfrak{p}_{+}$, and by $K \mathcal{P}$ the subspace of $K$-invariant polynomials. Now let $\mathcal{H}=\mathcal{H}\left(\mathfrak{p}_{+} \times \mathfrak{p}_{+}\right)$be 
the set of polynomials $q(z, w)$ on $\mathfrak{p}_{+} \times \mathfrak{p}_{+}$holomorphic in $z$ and antiholomorphic in $w$, satisfying the symmetry condition

$$
\overline{q(w, z)}=q(z, w)
$$

If $h \in \mathcal{H}$, then $z \longmapsto q(z, z)$ defines a real-valued polynomial $Q$ on $\mathfrak{p}_{+}$, and any realvalued polynomial $Q$ on $\mathfrak{p}^{+}$is obtained from a unique element $q$ of $\mathcal{H}$ by this process. If $Q$ is $K$-invariant, then $q$ is $K$-invariant on $\mathfrak{p}_{+} \times \mathfrak{p}_{+}$, because the action of $K$ on $\mathfrak{p}_{+}$is holomorphic and commutes with the conjugation $\sigma$, thus preserving $\mathcal{H}$. So the space $K \mathcal{P}$ is isomorphic to $K \mathcal{H}$, the space of $K$-invariant elements in $\mathcal{H}$. The same construction can be used for $\mathfrak{p}_{+}^{T}$, thus giving isomorphisms between $\mathcal{P}^{T}$ and $\mathcal{H}^{T}$ one one hand and $K_{T} \mathcal{P}^{T}$ and $K_{T} \mathcal{H}^{T}$ on the other (with obvious notation).

Lemma 1.2. Let $q \in K \mathcal{P}$, and $Q \in K \mathcal{H}$ the associated element. Let $q^{T}$ the element of $K_{T} \mathcal{P}^{T}$ whose restriction to $\mathfrak{a}_{+}$is $q_{\left.\right|_{\mathfrak{a}_{+}}}$, and $Q^{T}$ the associated element in $K_{T} \mathcal{H}^{T}$. Then, for any $z^{T} \in \mathfrak{p}_{+}^{T}$ and $w \in \mathfrak{p}_{+}$,

$$
Q\left(z^{T}, w\right)=Q^{T}\left(z^{T}, P w\right)
$$

where $P$ is the projection of $\mathfrak{p}_{+}$on $\mathfrak{p}_{+}^{T}$ with respect to eigenspaces of the involution $\operatorname{Ad}\left(c^{4}\right)$.

Proof. Although not stated, the lemma is a consequence of the results obtained in [F-al] part III, sections V and VI. We use freely the notation from [F-al]. Let $E_{\Gamma}=\sum_{j=1}^{r} E_{j}$. This is an element in $\mathfrak{p}_{+}^{T}$, such that the orbit $S$ (resp. $S^{T}=S \cap \mathfrak{p}_{+}^{T}$ ) of $E_{\Gamma}$ under $K$ (resp. $K^{T}$ ) is the Shilov boundary of $\mathcal{D}$ (resp. $\mathcal{D}^{T}$ ). Let $L$ (resp. $L^{T}$ ) be its stabilizer in $K$ (resp. $K^{T}$ ). Then consider the function defined for $w \in \mathfrak{p}_{+}$ by $\varphi(w)=Q\left(E_{\Gamma}, w\right)$. This is an anti-holomorphic polynomial on $\mathfrak{p}_{+}$, invariant by $L$. Hence it is a (finite) linear combination of the $L$-invariant polynomials $\left\{\varphi_{\mathbf{m}}(w)\right\}$ and hence

$$
Q\left(E_{\Gamma}, w\right)=Q^{T}\left(E_{\Gamma}, P w\right)
$$

(see theorem V.2.1 and lemma VI.4.5 in [F-al] Part III ). Now by $K^{T}$-invariance, and the fact that the action of $K^{T}$ commutes with the projection $P$, we easily get

$$
Q\left(k E_{\Gamma}, w\right)=Q^{T}\left(k E_{\Gamma}, P w\right)
$$

for $k \in K^{T}$. As the orbit of $E_{\gamma}$ under $K^{T}$ is the Shilov boundary $S^{T}$ of $\mathcal{D}^{T}$,

$$
Q(\sigma, w)=Q^{T}(\sigma, P w)
$$

for $\sigma \in S^{T}$. As $Q\left(z^{T}, w\right)$ and $Q^{T}\left(z^{T}, P w\right)$ are holomorphic in $z^{T}$, they must coincide everywhere on $\mathcal{D}^{T}$, hence on $\mathfrak{p}_{+}^{T}$.

Applied to the invariant polynomial $h^{2}$, we obtain the following formula

$$
k\left(z^{T}, w\right)=k^{T}\left(z^{T}, P w\right)
$$

for all $z^{T} \in \mathfrak{p}_{+}^{T}$ and $w \in \mathfrak{p}_{+}$. 
Proposition 1.3. Let $z \in \mathcal{D}$. Then $P z \in \mathcal{D}^{T}$.

Proof. Consider on $\mathfrak{g}_{\mathbb{C}}$ the (standard) Hermitian inner product given by

$$
<X, Y>=-B(X, \sigma \theta Y>
$$

where $B$ is as before the Killing form of $\mathfrak{g}$ extended by $\mathbb{C}$-linearity to $\mathfrak{g}_{\mathbb{C}}$ and for $z \in \mathfrak{p}^{+}$, let $\Re z=\frac{1}{2}(z+\sigma(z)) \in \mathfrak{p}$. Then the domain $\mathcal{D}$ admits the following description

$$
\mathcal{D}=\left\{z \in \mathfrak{p}_{+} \mid\|\operatorname{ad} \Re z\|<1\right\}
$$

where the norm refers to the operator norm on $\operatorname{End}\left(\mathfrak{g}_{\mathbb{C}}\right)$ (see $[S]$ ch. II, Prop. 4.6). There is of course a similar description for $\mathcal{D}_{T}$, namely

$$
\mathcal{D}_{T}=\left\{z \in \mathfrak{p}_{+}^{T} \mid\left\|\operatorname{ad}_{\mid \mathfrak{g}_{\mathbb{C}}^{T}} \Re z\right\|_{T}<1\right\} \text {. }
$$

Here the norm is the operator norm on $\operatorname{End}\left(\mathfrak{g}_{T}\right)$, where $\mathfrak{g}_{T}$ is equipped with the standard inner product $<., .>_{T}$ coming from the Killing form $B_{T}$ of $\mathfrak{g}_{\mathbb{C}}^{T}$. The following lemma gives the relation between the two inner products.

LEMMA 1.4. The restriction of the inner product $<., .>$ to $\mathfrak{g}^{T}$ is a (non-zero) multiple of $<.,.\rangle_{T}$.

The space $\mathfrak{a}$ is a Cartan subspace for both pairs $\mathfrak{g}, \mathfrak{k}$ and $\mathfrak{g}^{T}, \mathfrak{k}^{T}$. The set $\left\{X_{1}, X_{2}, \ldots, X_{r}\right\}$ is an orthogonal basis for both inner products, and we showed that $B\left(X_{j}, X_{j}\right)=4 p$. The same computation can be done for $\mathfrak{g}^{T}$ and gives $B^{T}\left(X_{j}, X_{j}\right)=$ $4 p_{T}$ where $p_{T}=(r-1) a+2$. Hence the two inner product are proportional on $\mathfrak{a}$. By $K_{T}$ invariance, they are proportional on $\mathfrak{p}^{T}$, and hence on $\mathfrak{g}_{T}$ as $\mathfrak{g}_{T}$ has no compact factor.

The lemma allows us to compute the operator norm on $\mathfrak{g}_{\mathbb{C}}^{T}$ by using the inner product $<.$, . $>$.

Now consider the decomposition of $\mathfrak{g}$ with respect to $\operatorname{Ad}\left(c^{4}\right)$

$$
\mathfrak{g}=\mathfrak{g}^{T} \oplus \mathfrak{g}^{(2)}, \quad \mathfrak{k}=\mathfrak{k}^{T} \oplus \mathfrak{q}^{(2)}, \quad \mathfrak{p}=\mathfrak{p}^{T} \oplus \mathfrak{p}^{(2)}
$$

and let $X=X^{T}+X^{(2)}$ be the decomposition of an element in $\mathfrak{p}$. Let $Y \in \mathfrak{g}_{\mathbb{C}}^{T}$. Then

$$
\operatorname{ad} X(Y))=\left[X^{T}, Y\right]+\left[X^{(2)}, Y\right]
$$

But $\left[X^{T}, Y\right]$ belongs to $\mathfrak{g}_{\mathbb{C}}^{T}$ and $\left[X^{(2)}, Y\right]$ belongs to $\mathfrak{g}^{(2)}$. As $\mathfrak{g}_{\mathbb{C}}^{T}$ and $\mathfrak{g}_{\mathbb{C}}^{(2)}$ are orthogonal, we get

$$
\left\|\operatorname{ad}\left(X^{T}\right) Y\right\| \leq\|\operatorname{ad}(X) Y\| .
$$

Assume now that $X=\Re z$ for some $z \in \mathcal{D}$. Then $\|\operatorname{ad}(X) Y\|<\|Y\|$ and hence

$$
\left\|\operatorname{ad}\left(X^{T}\right) Y\right\|<\|Y\| \text {. }
$$

As $Y$ is arbitrary in $\mathfrak{g}_{\mathbb{C}}^{T}$ we get $\left\|\operatorname{ad}\left(X^{T}\right)\right\|<1$. But the projection on $\mathfrak{p}_{+}^{T}$ commutes with "taking the real part", so that we get $\|\operatorname{ad}(\Re P z)\|<1$, proving that $P z \in \mathcal{D}^{T}$. 


\section{Proposition 1.5. Let $\sigma \in S$ and assume that $P \sigma \in S^{T}$. Then $\sigma \in S^{T}$.}

Proof. As $K^{T}$ acts transitively on $S^{T}$ we may assume that $P \sigma=E=\sum_{j=1}^{r} E_{j}$. Let $L$ be the real affine hyperplane through $E$ which is orthogonal to the line $\mathbb{R} E$. Then one has $\overline{\mathcal{D}} \cap L=\{E\}$. Now $L$ contains $E+\mathfrak{p}_{+}^{(2)}$, so that no point of $E+\mathfrak{p}_{+}^{(2)}$ can belong to $S$ except $E$.

2. Computation of the integrals $\int_{\Delta} \omega$. The Hermitian symmetric space $\mathcal{D}$ is a Kaehler manifold. Its (normalized) Kaehler form $\omega$ is given by

$$
\omega=i \partial \bar{\partial} \log k(z, z) \text {. }
$$

see $([\mathrm{S}])$. It is a closed 2 -form of type $(1,1)$. If $\Delta$ is a geodesic triangle (i.e. the sides of which are geodesic segments) in $\mathcal{D}$, we may consider any smooth surface $\Sigma$ which has the geodesic triangle as its boundary. As $\omega$ is closed, $\int_{\Sigma} \omega$ does not depend on $\Sigma$, but merely on $\Delta$. Hence we denote this integral by $\int_{\Delta} \omega$, and call it occasionally the symplectic area of the (geodesic) triangle $\Delta$.

TheOREm 2.1. Let $\Delta=\left(w_{1}, w_{2}, w_{3}\right)$ be an (oriented) geodesic triangle in $\mathcal{D}$. Then

$$
\int_{\Delta} \omega=\varphi\left(w_{1}, w_{2}, w_{3}\right)=-\left(\arg k\left(w_{1}, w_{2}\right)+\arg k\left(w_{2}, w_{3}\right)+\arg k\left(w_{3}, w_{1}\right)\right) .
$$

where $\arg k(z, w)$ is the (unique) continuous determination of the argument such that $\arg k(z, z)=0$ for any $z \in \mathcal{D}$.

For the proof we follow $[\mathrm{D}-\mathrm{T}]$ and use Stokes formula. This is better explained by introducing the operator $d_{c}$ on differential forms (see e.g. [Gol] section 2.4). Let $J$ denote the complex structure operator in the tangent bundle. It induces an operator on the cotangent bundle (still denoted by $J$ ) defined by

$$
J \xi(X)=\xi(J X)
$$

which has then a natural extension to the exterior bundle. On forms of type $(p, q)$ it coincides with $i(q-p)$. The operator $d_{c}$ is defined by

$$
d_{c} \varphi=J^{-1} d J \varphi
$$

where $\varphi$ is a differential form. Then $d_{c}=-i(\partial-\bar{\partial}), d d_{c}=2 i \partial \bar{\partial}$. For a holomorphic function $f$ on $\mathcal{D}$, the Cauchy-Riemann equations have the following equivalent formulation

$$
d_{c} \Re f=d \Im f \quad d_{c} \Im f=-d \Re f .
$$

The computation uses appropriate potentials for the Kaehler form. Let us first consider the base-point $o$. Then set

$$
\rho_{o}(z)=\log k(z, z)
$$

Proposition 2.2. The function $\rho_{o}$ satisfies the following properties
i) $d d_{c} \rho_{o}=2 \omega$.
ii) $\rho_{o}(o)=0$ 
iii) $\rho_{o}$ is invariant under $K$

$i v) d_{c} \rho_{o}$ vanishes on tangent vectors to any geodesic through $o$.

Let us only prove $i v$ ) since the other properties are obvious. Let us consider a geodesic $t \mapsto \gamma(t)$ which for $t=0$ passes through $o$. By $K$-invariance, we may assume that its tangent vector $\dot{\gamma}(0)$ at $o$ is in $\mathfrak{a}$. Let assume first that $\dot{\gamma}(0)$ is regular, i.e. $\alpha(\dot{\gamma}(0) \neq 0$ for all restricted roots $\alpha$. As $\mathfrak{a}$ is flat, the geodesic will remain in exp $\mathfrak{a}$ and $\gamma(t)$ is for every $t \neq 0$ a regular point in expa. Now if $X \in \mathfrak{a}$, then $J X$ is orthogonal to $\mathfrak{a}$ as

$$
B(J X, Y)=B\left(\left[H_{0}, X\right], Y\right)=B\left(H_{0},[X, Y]\right)=0
$$

for any $Y \in \mathfrak{a}$. Hence $J \dot{\gamma}(t) \in \mathfrak{a}^{\perp}$. But at the regular point $m=\gamma(t), t \neq 0$, the orthogonal of $\mathfrak{a}$ in the tangent space at $m$ is exactly the tangent space to the $K$-orbit at $m$. Hence $i i i)$ implies that $d \rho_{0}(J \dot{\gamma}(t))=0$ for any $t \in \mathbb{R}$. The case of a non regular geodesic is obtained by continuity.

Now let $w \in \mathcal{D}$. Let $g \in G$ such that $w=g(0)$. Then define

$$
\rho_{w}(z)=\rho_{0}\left(g^{-1} z\right)
$$

Observe that the definition does not depend on the choice of $g$. By invariance under $G$ of the Kaehler form, the function $\rho_{w}$ satisfies

i) $d d_{c} \rho_{w}=2 \omega$.

ii) $\rho_{w}(w)=0$

iii) $\rho_{w}$ is invariant under the stabilizer of $w$ in $G$

$i v) d_{c} \rho_{w}$ vanishes on tangent vectors to any geodesic through $w$.

Now, from (1.3)

$$
\rho_{w}(z)=\rho_{0}(z)-\frac{4}{p} \log \left|j\left(g^{-1}, z\right)\right|
$$

hence

$$
\rho_{0}(z)-\rho_{w}(z)=\frac{4}{p} \log \left|j\left(g^{-1}, z\right)\right|=\frac{4}{p} \Re \log j\left(g^{-1}, z\right) .
$$

We now use a specific element $g$, namely $g_{w}$ (see definition in section 1). First observe that as $g_{w} \in G, \sigma\left(g_{w}\right)=g_{w}$, and so

$$
g_{w}^{-1}=\exp (-w) K(w, w)^{\frac{1}{2}} \exp (-\sigma(w)) .
$$

Hence

$$
g_{w}^{-1} \exp (z)=\exp (-w) K(w, w)^{\frac{1}{2}} p_{+} K(z, w)^{-1} p_{-},
$$

with $p_{+} \in P_{+}, p_{-} \in P_{-}$. As $K_{\mathbb{C}}$ normalizes $P_{+}$, we get

$$
J\left(g_{w}^{-1}, z\right)=K(w, w)^{\frac{1}{2}} K(z, w)^{-1}
$$

and hence

$$
j\left(g_{w}^{-1}, z\right)=k_{\mathcal{D}}(w, w)^{-\frac{1}{2}} k_{\mathcal{D}}(z, w)
$$

or

$$
j\left(g_{w}^{-1}, z\right)^{\frac{4}{p}}=k(w, w)^{-1} k(z, w)^{2}
$$


Now let $\Delta$ be a geodesic triangle with summits $0, w_{1}, w_{2}$. Then, by Stokes formula

$$
\int_{\Delta} \omega=\frac{1}{2} \int_{\Delta} d d_{c} \rho_{0}=\frac{1}{2} \int_{\gamma_{w_{1}, w_{2}}} d_{c} \rho_{0}
$$

where $\gamma_{w_{1}, w_{2}}$ denotes the geodesic arc from $w_{1}$ to $w_{2}$. Now, thanks to $(2.5)$

$$
\int_{\gamma_{w_{1}, w_{2}}} d_{c} \rho_{0}=\int_{\gamma_{w_{1}, w_{2}}} d_{c} \rho_{w}+\int_{\gamma_{w_{1}, w_{2}}} d_{c}\left(\frac{4}{p} \Re \log j\left(g^{-1}, z\right)\right) .
$$

The first integral in the right hand-side vanishes because of property $i v$ ), and the second integral can be transformed using the Cauchy-Riemann equations for the holomorphic function $j\left(g^{-1}, z\right)$, to yield

$$
\begin{gathered}
\int_{\Delta} \omega=\int_{\gamma_{w_{1}, w_{2}}} d\left(\frac{2}{p} \Im \log j\left(g^{-1}, z\right)\right)=\frac{2}{p}\left(\arg j\left(g^{-1}, w_{2}\right)-\arg j\left(g^{-1}, w_{1}\right)\right) \\
=\arg k\left(w_{2}, w_{1}\right)=-\arg k\left(w_{1}, w_{2}\right) .
\end{gathered}
$$

To finish the proof ot Theorem 1, we first observe that $k(o, w)=k(w, o)=1$, so that the formula for the geodesic triangle $\left(o, w_{1}, w_{2}\right)$ corresponds to the statement of the theorem. Then we conclude by observing that both sides of the formula are invariant by the action of $G$, so that it is enough to prove it for triangle with first summit equal to $o$.

3. Estimate of the integrals $\int_{\Delta} \omega$.

TheOREM 3.1. For any geodesic triangle $\Delta$

$$
\left|\int_{\Delta} \omega\right|<r \pi
$$

REMARK. The estimate is optimal in the sense that

$$
\sup _{\Delta} \int_{\Delta} \omega=r \pi, \inf _{\Delta} \int_{\Delta} \omega=-r \pi \text {. }
$$

where $\Delta$ runs through all geodesic triangles in $\mathcal{D}$.

The proof is divided in several steps.

Step 1. Reduction to the tube-type case. As $G$ is transitive on $\mathcal{D}$, we may always assume that the first summit $z_{1}$ of the triangle is $o$. Next by using the action of $K$, we may assume that the second summit $z_{2}$ is in $\mathfrak{a}_{+}$, so in particular $z_{2} \in \mathfrak{p}_{+}^{T}$. Thanks to Lemma 1.2 , we loose no generality in assuming that the third summit is in $\mathfrak{p}_{+}^{T}$.

Step 2. Reduction to the Shilov boundary. Assume that we are in the tube-type case, and so we may drop the upper-index $T$. The value of the integral as expressed by formula (2.2) coincides (up to a constant) with the expression used in [C- $\varnothing]$ to generalize the Maslov index. More precisly, in [C- $\varnothing]$ we set

$$
c\left(w_{1}, w_{2}, w_{3}\right)=\frac{k\left(w_{1}, w_{2}\right)}{k\left(w_{2}, w_{1}\right)} \frac{k\left(w_{2}, w_{3}\right)}{k\left(w_{3}, w_{2}\right)} \frac{k\left(w_{3}, w_{1}\right)}{k\left(w_{1}, w_{3}\right)}
$$


so that

$$
\varphi\left(w_{1}, w_{2}, w_{3}\right)=-\frac{1}{2} \arg \left(c\left(w_{1}, w_{2}, w_{3}\right)\right) .
$$

We were interested in the limit of these expressions when the summits approach the Shilov boundary to form an ideal triangle with summits in $S$. The estimate which we are looking for is obtained along the same line. First let $0<t<1$. Then consider the auxiliary function $\varphi_{t}$ defined by

$$
\varphi_{t}\left(w_{1}, w_{2}, w_{3}\right)=-\left(\arg \left(k\left(t w_{1}, t w_{2}\right)+\arg k\left(t w_{2}, t w_{3}\right)+\arg k\left(t w_{3}, t w_{1}\right)\right) .\right.
$$

As a function of $w_{1}$, it is a sum of the real part of a holomorphic function and the real part of an anti-holomorphic function, hence it is a pluri-harmonic function. Of course the definition of $\varphi_{t}$ can be extended to a neighbourhood of $\overline{\mathcal{D}}$. The maximum principle for holomorphic functions with respect to the Shilov boundary $S$ extends to pluri-harmonic functions. In fact, such a function (say $f$ ) is the real part of some holomorphic function (say $F$ ), so $e^{f}$ is the modulus of the holomorphic function $e^{F}$, and $e^{f}$ (hence also $f$ ) reaches its maximum on $S$. We get

$$
\sup _{w_{1}, w_{2}, w_{3} \in \mathcal{D}}\left|\varphi_{t}\left(w_{1}, w_{2}, w_{3}\right) \leq \sup _{\omega_{1} \in S, w_{2}, w_{3} \in \mathcal{D}}\right| \varphi_{t}\left(\omega_{1}, w_{2}, w_{3}\right) \mid
$$

We may apply the same argument to $w_{2}$ and then $w_{3}$ to get

$$
\sup _{w_{1}, w_{2}, w_{3} \in \mathcal{D}}\left|\varphi_{t}\left(w_{1}, w_{2}, w_{3}\right)\right| \leq \sup _{\omega_{1}, \omega_{2}, \omega_{3} \in \mathcal{S}}\left|\varphi_{t}\left(\omega_{1}, \omega_{2}, \omega_{3}\right)\right|
$$

As $\varphi_{t}$ is continuous on $S \times S \times S$, it is enough to estimate it on a dense open subset. Hence we may use the set $S_{\top}^{3}$ of triplets of mutually transverse elements (see [C- $\left.\varnothing\right]$ for the definition).

Step 3. As a function of $t$, the function $\sup _{S \times S \times S}\left|\varphi_{t}\right|=\sup _{t \mathcal{D} \times t \mathcal{D} \times t \mathcal{D}}|\varphi|$ is increasing. But for $\left(\omega_{1}, \omega_{2}, \omega_{3}\right) \in S_{\top}^{3}, \varphi_{t}\left(\omega_{1}, \omega_{2}, \omega_{3}\right)$ has a limit as $t \rightarrow 1$. In fact a special case of the results in $[\mathrm{C}-\varnothing]$ gives that

$$
\frac{1}{2 \pi} \arg c\left(t \omega_{1}, t \omega_{2}, t \omega_{3}\right) \longrightarrow \iota\left(\omega_{1}, \omega_{2}, \omega_{3}\right)
$$

where $\iota\left(\omega_{1}, \omega_{2}, \omega_{3}\right)$ is the Maslov index of the triple $\left(\omega_{1}, \omega_{2}, \omega_{3}\right)$, an integer between $r$ and $-r$. Hence $\left|\varphi_{t}\left(\omega_{1}, \omega_{2}, \omega_{3}\right)\right| \leq r \pi$. As $t$ is arbitrary in $] 0,1[$, the inequality $\left|\int_{\Delta} \omega\right| \leq r \pi$ follows. The inequality is strict. It this were not the case, then the pluri-harmonic function $\varphi$ would reach its maximum (or infimum) in the interior of $\mathcal{D}^{3}$, so would be constant on $\mathcal{D}^{3}$, which is obviously not true.

Actually, the proof gives a little more, namely that

$$
\sup _{\Delta}\left|\int_{\Delta} \omega\right|=r \pi
$$

where $\Delta$ runs through all geodesic triangles in $\mathcal{D}$.

In fact, it is enough to prove this property for tube-type domains. In this case, choose three points $\omega_{1}, \omega_{2}, \omega_{3}$ mutually tranverse in $S$ such that their Maslov index 
equals $r$. Then consider for $0<t<1$ the triangles $\Delta_{t}$ with summits $\left(t \omega_{1}, t \omega_{2}, t \omega_{3}\right)$. Clearly they satisfy

$$
\limsup _{t \rightarrow 1} \int_{\Delta_{t}} \omega=r \pi .
$$

This question will be further discussed at the end of section 4 .

4. Ideal triangles. Let $\xi_{1}, \xi_{2}, \xi_{3}$ be three distinct points in the topological boundary $\partial \mathcal{D}$ of $\mathcal{D}$, and assume there is an (infinite) geodesic with endpoints $\xi_{1}$ on one hand and $\xi_{2}$ on the other, and similarly for $\left(\xi_{2}, \xi_{3}\right)$ and $\left(\xi_{3}, \xi_{1}\right)$. This describes an ideal triangle.

If we consider two disctinct points of the boundary $\xi_{1}$ and $\xi_{2}$, there might exist no geodesic line with endpoints $\xi_{1}$ and $\xi_{2}$ or there might be several geodesics satisfying this condition. Recall that the topological boundary is a disjoint union of exactly $r$ orbits under the action of $G$ (see [S] Theorem 8.7). In fact let for $1 \leq k \leq r$

$$
E^{k}=\sum_{j=1}^{k} E_{j}
$$

Then $E^{k}$ belongs to $\partial \mathcal{D}$ and

$$
\partial \mathcal{D}=\bigsqcup_{k=1}^{r} \partial_{k} \mathcal{D}
$$

where $\partial_{k} \mathcal{D}=G \cdot E^{k}$.

Before stating our next result we need a new definition (already introduced in the Jordan algebra setting in [C- $\varnothing])$. If two elements $(z, w)$ of $\mathfrak{p}_{+}$satisfy $h(z, w) \neq 0$, we say that they are transverse and denote this relation by

$$
z \top w \Longleftrightarrow h(z, w) \neq 0 \text {. }
$$

Because of the properties of the kernel $h$, this relation is symmetric and $G$-invariant.

Proposition 4.1. Two points of $\partial \mathcal{D}$ which are endpoints of some (infinite) geodesic belong to the same $G$-orbit and are transverse.

Because of the transitivity of the action of $G$, we may always assume that the geodesic passes through the origin $o$, say at time 0 . Similarly by using known properties of the stabilizer $K$ of $o$, we may assume that its tangent vector $\dot{\gamma}(0)$ at $o$ is in a specific Cartan subspace and even in a dominant Weyl chamber. So we may assume w.l.o.g. that

$$
\dot{\gamma}(0)=\sum_{j=1}^{r} a_{j} E_{j}
$$

with $a_{1} \geq a_{2} \geq \ldots \geq a_{r} \geq 0$. Hence the geodesic is of the form

$$
t \mapsto \gamma(t)=\sum_{j=1}^{r} \tanh \left(a_{j} t\right) E_{j}
$$

Assume that $a_{k}>0$ but $a_{k+1}=a_{k+2}=\ldots=a_{r}=0$. Then

$$
\gamma(t) \longrightarrow \gamma(\infty)=E^{k} \quad \text { as } t \rightarrow+\infty, \quad \gamma(t) \longrightarrow \gamma(-\infty)=-E^{k} \quad \text { as } t \rightarrow-\infty .
$$


Clearly $\gamma(\infty)$ belongs to the same $G$-orbit as $\gamma(-\infty)$. To see the transversality relation, notice that by polarization of the definition of $h$, one as

$$
h\left(\sum_{j=1}^{r} s_{j} E_{j}, \sum_{j=1}^{r} t_{j} E_{j}\right)=\prod_{j=1}^{r}\left(1-s_{j} \overline{t_{j}}\right)
$$

for $s_{j}, t_{j} \in \mathbb{C}, 1 \leq j \leq r$. Hence $h(\gamma(\infty), \gamma(-\infty))=2^{k} \neq 0$.

By an ideal triangle of type $k$, we mean an ideal triangle $\Delta$ with summits in $\partial_{k} \mathcal{D}$.

For $A$ any subset of $\mathfrak{p}^{+}$, set

$$
A_{\top}^{3}=\left\{\left(a_{1}, a_{2}, a_{3}\right) \mid a_{j} \in A, a_{j} \top a_{k}, j \neq k\right\} .
$$

Now let

$$
\widetilde{\mathcal{D}}^{3}=(\overline{\mathcal{D}})_{\top}^{3} \supset \mathcal{D}^{3} \cup \bigsqcup_{k=1}^{r}\left(\partial_{k} \mathcal{D}\right)_{\top}^{3} .
$$

As $\mathcal{D}$ is star-shaped with respect to 0 , the set $\widetilde{\mathcal{D}}^{3}$ is clearly simply connected. Moreover the function

$$
\left(z_{1}, z_{2}, z_{3}\right) \longmapsto h\left(z_{1}, z_{2}\right) h\left(z_{2}, z_{3}\right) h\left(z_{3}, z_{1}\right)
$$

is continuous and does not vanish on $\widetilde{\mathcal{D}}^{3}$. Hence the $\mathbb{C}^{*}$-valued function

$$
\left(z_{1}, z_{2}, z_{3}\right) \longmapsto k\left(z_{1}, z_{2}\right) k\left(z_{2}, z_{3}\right) k\left(z_{3}, z_{1}\right)
$$

is well-defined and continous on $\widetilde{\mathcal{D}}^{3}$. So there is a well-defined continuous extension to $\widetilde{\mathcal{D}}^{3}$ of the function $\varphi$ which was used to compute the integral of the Kaehler form on a geodesic triangle. We will call it occasionally the triple ratio of the corresponding summits of the triangle.

If $\Delta$ is an ideal triangle, with summits (say) $\left(\xi_{1}, \xi_{2}, \xi_{3}\right)$, then $\left(\xi_{1}, \xi_{2}, \xi_{3}\right)$ belongs to $\widetilde{\mathcal{D}}^{3}$, and hence we may extend the definition of the symplectic area $\int_{\Delta} \omega$ by

$$
\int_{\Delta} \omega=\varphi\left(\xi_{1}, \xi_{2}, \xi_{3}\right)=\lim _{z_{j} \rightarrow \xi_{j}, z_{j} \in \mathcal{D}} \int_{\Delta\left(z_{1}, z_{2}, z_{3}\right)} \omega
$$

Notice in particular that $\int_{\Delta} \omega$ depends only on the summits of the ideal triangle $\Delta$ and not of the geodesic sides which are far from unique in general.

TheOREm 4.2. Let $\Delta$ be an ideal triangle of type $k$ where $1 \leq k \leq r$. Then

$$
\left|\int_{\Delta} \omega\right| \leq k \pi
$$

The case $k=r$ is an easy consequence of Theorem 3.1. For $k<r$, the proof uses a realization of the domain $\mathcal{D}$ as a Siegel domain of type III. We follow [S] except for minor changes of notation.

Let $k$ be an integer with $1 \leq k \leq r$,

$$
X^{\kappa}=\sum_{j=1}^{k} X_{j}, Y^{\kappa}=\sum_{j=1}^{k} Y_{j}, H^{\kappa}=\sum_{j=1}^{k} H_{j} .
$$


Then

$$
\mathfrak{s}=\mathfrak{s}^{\kappa}=\mathbb{R} X^{\kappa} \oplus \mathbb{R} Y^{\kappa} \oplus \mathbb{R} H^{\kappa}
$$

is a subalgebra of $\mathfrak{g}$ isomorphic to $\mathfrak{s l}_{2}(\mathbb{R})$ with the following relations

$$
\left[H^{\kappa}, X^{\kappa}\right]=-2 Y^{\kappa}, \quad\left[H^{\kappa}, Y^{\kappa}\right]=2 X^{\kappa}, \quad\left[X^{\kappa}, Y^{\kappa}\right]=2 H^{\kappa}
$$

Let $c_{\kappa}=\exp \left(i \frac{\pi}{4} Y_{\kappa}\right)$ be the "Cayley element" in $G_{\mathbb{C}}$ and let $\operatorname{Ad} c_{\kappa} \in \operatorname{Ad}\left(g_{\mathbb{C}}\right)$ to be

$$
\operatorname{Ad} c_{\kappa}=\operatorname{Ad}\left(\exp \left(i \frac{\pi}{4} Y_{\kappa}\right)\right)=\exp \left(\frac{i \pi}{4} \operatorname{ad} Y^{\kappa}\right)
$$

One has the following elementary results

$$
\operatorname{Ad} c_{\kappa}\left(H^{\kappa}\right)=-i X^{\kappa}, \quad \operatorname{Ad} c_{\kappa}\left(X^{\kappa}\right)=-i H^{\kappa}, \quad \operatorname{Ad} c_{\kappa}\left(Y^{\kappa}\right)=Y^{\kappa}
$$

On decomposing in irreducible components the representation (adjoint action) of $\mathfrak{s}$ into $\mathfrak{g}$, only factors of dimension $1+d$ with $d=0,1,2$ may appear, so that the isotypic decomposition is (with obvious notation)

$$
\mathfrak{g}=\mathfrak{g}^{[0]} \oplus \mathfrak{g}^{[1]} \oplus \mathfrak{g}^{[2]} .
$$

The map $\operatorname{Ad} c_{\kappa}^{4}$ is an involution of $\mathfrak{g}$, and hence, the space of its fixed points $\mathfrak{g}^{\text {[even] }=}$ $\mathfrak{g}^{[0]} \oplus \mathfrak{g}^{[2]}$ is a Lie subalgebra. Consider its decomposition

$$
\mathfrak{g}^{[\text {even] }}=\mathfrak{g}_{0} \oplus \bigoplus_{i=1}^{s} \mathfrak{g}_{s}
$$

where $\mathfrak{g}_{0}$ is the largest compact ideal, and the $\mathfrak{g}_{i}(i \geq 1)$ are simple and non-compact ideals.

Let

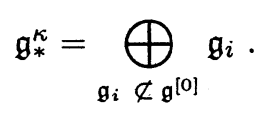

The Lie algebra $\mathfrak{g}_{*}^{\kappa}$ is semi-simple, Hermitian of the non-compact type, of tube type, with $H_{0}$-element $\frac{1}{2} H^{\kappa}$. It is stable by $\theta$. It has a Cartan decomposition

$$
\mathfrak{g}_{*}^{\kappa}=\mathfrak{g}_{*}^{\kappa} \cap \mathfrak{k} \oplus \mathfrak{g}_{*}^{\kappa} \cap \mathfrak{p}=\mathfrak{k}_{*}^{\kappa} \oplus \mathfrak{p}_{*}^{\kappa}
$$

The complexification $\left(p_{*}^{\kappa}\right)_{\mathbb{C}}$ decomposes under the action of the complex structure as $\mathfrak{p}_{\mathbb{C}}^{\kappa, *}=\mathfrak{p}_{*,+}^{\kappa} \oplus \mathfrak{p}_{*,-}^{\kappa}$. The corresponding symmetric space $G_{*}^{\kappa} / K_{*}^{\kappa}$ can be realized à la Harish Chandra as a bounded open set in $\mathfrak{p}_{*,+}^{\kappa}$ and appears as $\mathcal{D}_{*}^{\kappa}=\mathcal{D} \cap \mathfrak{p}_{*,+}^{\kappa}$. The space $\bigoplus_{j=1}^{k} \mathbb{R} Y_{k}$ is a Cartan subspace of the pair $\left(\mathfrak{g}_{*}^{\kappa}, \mathfrak{k}_{*}^{\kappa}\right)$. This implies in particular that the normalized Bergman kernel $k^{\kappa}$ of $\mathcal{D}_{*}^{\kappa}$ is the restriction of the (normalized) Bergman kernel of $\mathcal{D}$.

Let now

$$
\mathfrak{g}_{(2)}^{\kappa}=\left(\operatorname{Ad} c_{\kappa}^{-1}\left(\mathfrak{k}_{*}^{\kappa}\right)\right)_{\mathbb{C}} \cap \mathfrak{g} .
$$

It is contained in the centralizer of $X^{\kappa}$. Let us denote by $G_{(2)}^{\kappa}$ the analytic subgroup of $G$ with Lie algebra $\mathfrak{g}_{(2)}^{\kappa}$. 
Further, consider the Lie algebra

$$
\mathfrak{g}_{(1)}^{\kappa}=\bigoplus_{\mathfrak{g}_{i} \subset \mathfrak{g}^{[0]}} \mathfrak{g}_{i}, \quad \mathfrak{g}^{\kappa}=\bigoplus_{i \geq 1, \mathfrak{g}_{i} \subset \mathfrak{g}^{[0]}} \mathfrak{g}_{i}
$$

The Lie algebra $\mathfrak{g}^{\kappa}$ is semi-simple, Hermitian of the non-compact type, with $H_{0}$ element equal to $H_{0}-\frac{1}{2} H^{\kappa}$. As it is $\theta$-stable, we have

$$
\mathfrak{g}^{\kappa}=\mathfrak{g}^{\kappa} \cap \mathfrak{k} \oplus \mathfrak{g}^{\kappa} \cap \mathfrak{p}=\mathfrak{k}^{\kappa} \oplus \mathfrak{p}^{\kappa}
$$

and the complexification $\mathfrak{p}_{\mathbb{C}}^{\kappa}$ splits as

$$
\mathfrak{p}_{\mathbb{C}}^{\kappa}=\mathfrak{p}_{+}^{\kappa} \oplus \mathfrak{p}_{-}^{\kappa}
$$

Let $G^{\kappa}$ the analytic subgroup of $G$ with Lie algebra $\mathfrak{g}^{\kappa}$, and let $\mathcal{D}^{\kappa}=G^{\kappa} . o \subset \mathfrak{p}_{+}^{\kappa}$ its associated symmetric space.

Let

$$
\mathfrak{c}\left(X^{\kappa}\right)=\mathfrak{g}\left(\operatorname{ad} X^{\kappa} ; 0\right), \quad U^{\kappa}=\mathfrak{g}\left(\operatorname{ad} X^{\kappa} ; 2\right), \quad V^{\kappa}=\mathfrak{g}\left(\operatorname{ad} X^{\kappa} ; 1\right)
$$

and

$$
\mathfrak{b}^{\kappa}=\mathfrak{c}\left(X^{\kappa}\right) \oplus U^{\kappa} \oplus V^{\kappa}
$$

which is a parabolic subalgebra of $\mathfrak{g}$. One has

$$
\mathfrak{c}\left(X^{\kappa}\right)=\mathfrak{g}_{(1)}^{\kappa} \oplus \mathfrak{g}_{(2)}^{\kappa} .
$$

On the space $U^{\kappa}$ we define an inner product by

$$
<u, u^{\prime}>=-B\left(u, \theta u^{\prime}>, u, u^{\prime} \in U^{\kappa}\right.
$$

where $B$ is the Killing form of $\mathfrak{g}$. The group $G_{(1)}^{\kappa}$ operates trivially on $U^{\kappa}$. The element $E^{\kappa}=\frac{1}{2}\left(Y^{\kappa}+H^{\kappa}\right)$ belongs to $U^{\kappa}$ and $G_{(2)}^{\kappa} . E^{\kappa}=\Omega^{\kappa}$ is a self-adjoint homogeneous cone in $U^{\kappa}$ for the inner product we have defined. In fact, $G_{(2)}^{\kappa}$ is (the connected component of) the group of linear transformations of $U^{\kappa}$ which preserve $\Omega^{\kappa}$.

On $V^{\kappa}$ there is a complex structure $I_{0}$ (given by $\operatorname{ad}_{V^{\kappa}} 2\left(H_{0}-\frac{1}{2} H^{\kappa}\right.$ ), and an alternating bilinear map

$$
A: V^{\kappa} \times V^{\kappa} \longrightarrow U^{\kappa} \quad A\left(v, v^{\prime}\right)=-\frac{1}{4}\left[v, v^{\prime}\right]
$$

such that the bilinear form $\left(v, v^{\prime}\right) \longmapsto A\left(v, I_{0} v^{\prime}\right)$ is symmetric and $\Omega^{\kappa}$-positive definite in the sense that $A\left(v, I_{0} v\right) \in \bar{\Omega} \backslash\{0\}$ for all $v \in V^{\kappa}, v \neq 0$. The group $G_{(1)}^{\kappa}$ acts on $V^{\kappa}$ and preserves the bilinear map $A$, whereas the action of $G_{(2)}^{\kappa}$ on $V^{\kappa}$ commmutes with the complex structure $I_{0}$.

At this point, we will drop the index $\kappa$ most of the time, so that we set $V=$ $V^{\kappa}, U=U^{\kappa}$ and so on. The complexification $V^{\mathbb{C}}$ splits as

$$
V_{\mathbb{C}}=V_{+} \oplus V_{-}
$$


and to the alternating map $A$ we may associate the Hermitian map

$$
H: V_{+} \times V_{+} \rightarrow U_{\mathbb{C}}, \quad H\left(v, v^{\prime}\right)=i A\left(\bar{v}, v^{\prime}\right)
$$

which is moreover $\Omega$-positive in the sense that

$$
H(v, v) \in \bar{\Omega} \backslash\{0\} \text { for } v \in V_{+}, v \neq 0 .
$$

Finally, to each $t \in \mathcal{D}^{\kappa}$ there is a certain non-degenerate quasi-hermitian map (= sum of a Hermitian map and a $\mathbb{C}$-bilinear map) $\mathcal{L}_{t}: V_{+} \times V_{+} \longrightarrow U_{\mathbb{C}}$ which definition will not be needed explicitly, except for $t=o$, in which case

$$
\mathcal{L}_{o}\left(w, w^{\prime}\right)=H\left(w, w^{\prime}\right), \quad v, v^{\prime} \in V_{+} .
$$

Proposition 4.3. The holomorphic action of $c_{\kappa}$ is defined for all $z \in \mathcal{D}$ and the map $\left(\operatorname{Ad} c_{\kappa}\right)^{-1} c_{\kappa}$ gives an analytic isomorphism of $\mathcal{D}$ on the Siegel domain of the third kind $\mathcal{S}^{\kappa}$ defined by

$$
\mathcal{S}^{\kappa}=\left\{(u, v, t) \in U_{\mathbb{C}} \times V_{+} \times \mathcal{D}^{\kappa} \mid \Phi(u, v, t)=\Im u-\Re \mathcal{L}_{t}(v, v) \in \Omega\right\} .
$$

One can define an action of $G$ on $\mathcal{S}^{\kappa}$ in such a way that $\left(\operatorname{Ad} c_{\kappa}\right)^{-1} c_{\kappa}$ is equivariant with respect to $G$.

The description of the full action of $G$ would require more notation and will not be needed explicitly in the sequel. We will rather give the description of the action of a certain subgroup, which acts transitively and by quasi-linear maps (i.e. affine on any fiber of $\pi^{\kappa}$, where $\pi^{\kappa}: \mathcal{S}^{\kappa} \longrightarrow \mathcal{D}^{\kappa}$ is the projection on the third factor). Let $B^{\kappa}$ be the analytic subgroup of $G$ with Lie algebra $\mathfrak{b}^{\kappa}$. It is the semi-direct product of the connected component (denoted by $G^{1}$ ) of $C\left(X^{\kappa}\right)$ (the centralizer of $X^{\kappa}$ in $\mathfrak{g}$ ) and a simply connected step-two nilpotent subgroup $\widetilde{V}$ associated by the exponential map to the Lie algebra whose underlying vector space is $U \oplus V$, with the Lie bracket :

$$
\left[u+v, u^{\prime}+v^{\prime}\right]=-4 A\left(v, v^{\prime}\right) \text {. }
$$

The subgroup $G^{1}$ is the almost direct product of $G_{(1)}^{\kappa} . G_{(2)}^{\kappa}$. We denote an element of $B^{\kappa}$ by $g=\left(a, b, g_{1}\right)$ with $a \in U, b \in V, g_{1} \in G^{1}$. Then the map $\Phi$ is equivariant under the action of $B^{\kappa}$ in the sense that

$$
\Phi\left(g(u, v, t)=g_{1} \Phi(u, v, t), \quad(u, v, t) \in \mathcal{S}^{\kappa}\right.
$$

and similarly, the map $\pi^{\kappa}$ is equivariant under the action of $B^{\kappa}$ in the sense that

$$
\pi^{\kappa}(g(u, v, t))=g_{1}(t)
$$

for $g=\left(a, b, g_{1}\right) \in G^{1}$.

Moreover, the action of $B^{\kappa}$ is transitive on $\mathcal{S}^{\kappa}$, the subgroup $G_{(2)}^{\kappa} \cdot \widetilde{V}$ is transitive on the fiber

$$
\mathcal{F}^{\kappa}=\left(\pi^{\kappa}\right)^{-1}(o)=\{(u, v, o) \mid \Im u-H(v, v) \in \Omega\}
$$


and stabilizes the boundary point $(0,0, o)$ which is the image of $E^{\kappa}$ by the Cayley transform.

Some further information on that realization is needed.

Proposition 4.4. The fiber $\mathcal{F}^{\kappa}$ is the union of all geodesics of the domain $\mathcal{S}^{\kappa}$ with endpoint $(0,0, o)$.

Proof. The image of the geodesic $s \mapsto \gamma(s)=\exp s X^{\kappa} O$ is given by

$$
\left(\operatorname{Ad} c_{\kappa}\right)^{-1} c_{\kappa}(\gamma(s))=\left(i e^{2 s} E^{\kappa}, 0, o\right)
$$

(see $[\mathrm{S}])$. It is a geodesic which passes through $\left(i E^{\kappa}, 0, o\right)$ with endpoint $(0,0, o)$. By the transitivity of the action of $G_{(2)}^{\kappa} . \widetilde{V}$ on $\mathcal{F} \kappa$, there is a geodesic with endpoint $(0,0, o)$ through any point of that fiber, so that $\mathcal{F}^{\kappa}$ is a union of such geodesics. Now let $z \in \mathcal{S}^{\kappa}$. The set $\left\{(0,0, t), t \in \mathcal{D}^{\kappa}\right.$ is a holomorphic boundary component, and hence there is a unique geodesic issued from $z$ that has its endpoint in $\mathcal{D}^{\kappa}$. Let $t$ be its endpoint. As $G^{1}$ permutes the fibers of the map $\pi^{\kappa}$, it is also true that there is a geodesic through any point of $\left(\pi^{\kappa}\right)^{-1}(t)$ with endpoint $t$. So if the (unique) geodesic from $z$ with endpoint in $\mathcal{D}^{\kappa}$ has $(0,0, o)$ as endpoint, then the geodesic lies in $\mathcal{F} \kappa$, and hence $z \in \mathcal{F}^{\kappa}$.

Remark. However the space $\mathcal{F} \kappa$ is not a totally geodesic subspace of $\mathcal{S}^{\kappa}$. It has a structure of "quasi-symmetric" Siegel domain of type II.

Denote by $k_{\mathcal{S}}$ the Begman kernel of the domain $\mathcal{S}^{\kappa}$.

Proposition 4.5. The restriction of the Bergman kernel $k_{\mathcal{S}}$ to $\mathcal{F}^{\kappa} \times \mathcal{F}^{\kappa}$ is given by

$$
k_{\mathcal{S}}\left((u, v, o),\left(u^{\prime}, v^{\prime}, o\right)\right)=c_{\kappa} \operatorname{det}\left(\frac{1}{2 i}\left(u-\overline{u^{\prime}}\right)-H\left(v, v^{\prime}\right)\right)^{-\nu_{\kappa}}
$$

where $\nu_{\kappa}=\frac{1}{k}\left(2 \operatorname{dim} U^{\kappa}+\operatorname{dim}_{\mathbb{C}} V^{+}\right)$and some $c_{\kappa}>0$.

Proof. The subgroup $G_{(2)}^{\kappa}$ is reductive with a one-dimensional center. The center is in fact the one-parameter subgroup generated by $X^{\kappa}$. Hence two characters on $G_{(2)}^{\kappa}$ agree if and only if they agree on $\left\{\exp \left(\ln t \operatorname{ad} X^{\kappa}\right)\right\}_{t \in \mathbb{R}}$. Now,

$$
\exp \left(\ln t \operatorname{ad} X^{\kappa}\right)_{\mid U}=t^{2} \operatorname{Id}_{U}
$$

and

$$
\exp \left(\ln t \operatorname{ad} X^{\kappa}\right)_{\mid V}=t \operatorname{Id}_{V}
$$

Also recall that $G_{(2)}^{\kappa} . \tilde{V}$ operates transitively on $\mathcal{F}^{\kappa}$.

Consider the two functions on $\mathcal{F} \kappa$

$$
\left.(u, v, 0) \mapsto k_{\mathcal{S}}((u, v, 0),(u, v, 0)), \quad(u, v, 0)\right) \mapsto \operatorname{det}(\Im u-H(v, v))^{-\nu_{\kappa}}
$$

They are both invariant under the action of $\tilde{V}$. They both transform by a character under the action of $G_{(2)}^{\kappa}$ : the first one because of the transformation rule of the Bergman kernel under the (linear) action of $G_{(2)}^{\kappa}$ on $U_{\mathbb{C}} \times V^{+}$, the second because of 
the property of the function det in the Jordan algebra $U$. By looking to the action of the center of $G_{(2)}^{\kappa}$, we see that the exponent $-\nu_{\kappa}$ is precisely chosen so that both functions transform under the same character of $G_{(2)}^{\kappa}$. Hence the two functions coincide on $\mathcal{F} \kappa$. Finally the two expressions in the proposition are holomorphic in $(u, v)$ and anti-holomorphic in $\left(u^{\prime}, v^{\prime}\right)$, coincide when $(u, v)=\left(u^{\prime}, v^{\prime}\right)$ and hence are equal.

Denote by $\mathcal{T}=U+i \Omega \subset U_{\mathbb{C}}$ the tube domain associated to the Jordan algebra $U$, which we view as a subdomain of $\mathcal{F}$, and denote by $P_{\mathcal{T}}$ the projection from $U_{\mathbb{C}} \times V^{+}$ on $U_{\mathbb{C}}$.

Corollary 4.6. Let $z_{T} \in \mathcal{T}^{\kappa}$ and $z^{\prime} \in \mathcal{F}^{\kappa}$. Then

$$
k_{\mathcal{S}}\left(z_{T}, z^{\prime}\right)=k_{\mathcal{S}}\left(z_{T}, P_{\mathcal{T}} z^{\prime}\right) .
$$

Proof. It is a consequence of the formula (4.3), as one has to apply it with $v=v^{\prime}=0$. (1.5).

REMARK. In the case where $k=r$, this gives an independent proof of formula

We now come back to the proof of Theorem 4.2. Let $\Delta$ be an ideal triangle of type $k$. As we have already seen, it is possible to assume that one of the geodesic line is given by

$$
s \mapsto \gamma(s)=\exp s X^{\kappa}(o)=(\tanh s) E^{k} .
$$

There is a second geodesic $t \mapsto c(t)$ with endpoint at $c(-\infty)=-E^{k}$. In order to estimate the symplectic area of $\Delta$, it suffices to estimate the symplectic area of the geodesic triangle with summits $\gamma\left(s_{1}\right), \gamma\left(s_{2}\right), c(t)$ independently of $s_{1}, s_{2}$ and $t$. Now we use the Cayley transform : the corresponding summits say $\Gamma\left(s_{1}\right), \Gamma\left(s_{2}\right)$ and $C(t)$ are all three in the fiber $\mathcal{F}^{\kappa}$ as can be seen from Proposition 4.4, and the two first are in the domain $\mathcal{T} \kappa$. Hence from Corollary 4.6, we see that for the computation of the symplectic area of the geodesic triangle (which can be computed through the Bergman kernel) we may replace the third point $C(t)$ by its projection on $\mathcal{T}^{\kappa}$. In other words, we could assume from the beginning that the ideal triangle had its summits in the disc $\mathcal{D}_{*}^{\kappa}$. But now we use the estimate for the symplectic area of geodesic triangles in a tube-type domain of rank $k$ to conclude.

We now want to characterize the ideal triangles of maximal symplectic area. This is related to the notion of a holomorphic totally geodesic map from a hermitian symmetric space into another, which we now recall, following again [S]. Let $M, M^{\prime}$ be two Hermitian symmetric spaces of the non-compact type, $G, G^{\prime}$ their groups of biholomorphic diffeomorphisms, $\mathfrak{g}, \mathfrak{g}^{\prime}$ their Lie algebras. Let $\rho: M \rightarrow M^{\prime}$ be a holomorphic totally geodesic map. Fix $o \in M$ and choose $o^{\prime}=\rho(o)$ as origin in $M^{\prime}$. To the choice of these origins correspond Cartan decompositions

$$
\mathfrak{g}=\mathfrak{k} \oplus \mathfrak{p} \quad \mathfrak{g}^{\prime}=\mathfrak{k}^{\prime} \oplus \mathfrak{p}^{\prime}
$$

and corresponding $H_{0}$-elements, say $H_{0}$ and $H_{0}^{\prime}$. Then there always exists a homomorphism $\tilde{\rho}: \mathfrak{g} \longrightarrow \mathfrak{g}^{\prime}$ such that the following condition is satisfied :

$$
\tilde{\rho}\left(\left[H_{0}, X\right]\right)=\left[H_{0}^{\prime}, \tilde{\rho}(X)\right]
$$


for all $X \in \mathfrak{g}$. Moreover, the complex extension of $\tilde{\rho}$ induces a $\mathbb{C}$-linear map from $\mathfrak{p}_{+}$ into $\mathfrak{p}_{+}^{\prime}$ which maps $\mathcal{D}$ into $\mathcal{D}^{\prime}$ and coincides with $\rho: M \rightarrow M^{\prime}$ in the Harish Chandra realization of the Hermitian symmetric spaces $M$ and $M^{\prime}$ as bounded domains.

We will be interested in holomorphic totally geodesic imbeddings of the complex unit disc in $\mathcal{D}$. So set $\widetilde{\mathcal{D}}=\{z \in \mathbb{C}|| z \mid<1\}$ and let

$$
\widetilde{\mathfrak{g}}=\mathfrak{s u}(1,1)=\left\{\left(\begin{array}{cc}
i a & \beta \\
\bar{\beta} & -i a
\end{array}\right), a \in \mathbb{R}, \beta \in \mathbb{C}\right\} .
$$

Identify the complexification $\widetilde{\mathfrak{g}}_{\mathbb{C}}$ with $\mathfrak{s l}(2, \mathbb{C})$ and let $\widetilde{E}=\left(\begin{array}{ll}0 & 1 \\ 0 & 0\end{array}\right) \in \widetilde{\mathfrak{p}}_{+}$. Then there exists a choice of a Cartan subalgebra in $\mathfrak{k}$ and an integer $k, 1 \leq k \leq r$ such that $\rho(z)=z \sum_{j=1}^{k} E_{j}$. The imbedding will be called tight if $k=r$. It corresponds to a diagonal imbedding of the unit disc into the $r$-polydisc associated to any maximal (real) flat in the (Riemannian) symmetric space $\mathcal{D}$.

Let $\widetilde{\Delta}$ be any ideal triangle in $\widetilde{\mathcal{D}}$, with summits $\left\{\sigma_{1}, \sigma_{2}, \sigma_{3}\right\}$. Its image $\Delta=\rho(\widetilde{\Delta})$ under a tight (holomorphic totally geodesic) imbedding $\rho$ is an ideal triangle with summits $\left\{\left(\rho\left(\sigma_{1}\right), \rho\left(\sigma_{2}\right), \rho\left(\sigma_{3}\right)\right\}\right.$. It satisfies

$$
\int_{\Delta} \omega= \pm r \pi
$$

the sign depending on the orientation of $\widetilde{\Delta}$. In fact, by equivariance, it is enough to make the computation for the case where $\sigma_{1}=+1, \sigma_{2}=-1$ and $\sigma_{3}=-i$ (for ideal triangles of positive orientation). Using previous notation, we get $\rho\left(\sigma_{1}\right)=E^{r}, \rho\left(\sigma_{2}\right)=$ $-E^{r}$ and $\rho\left(\sigma_{3}\right)=-i E^{r}$. The computation of the triple ratio is now obvious (see also section 5 in $[\mathrm{C}-\varnothing])$.

Needless to say, any ideal triangle $\Delta^{\prime}$ with same summits as $\Delta$ will also satisfy $\int_{\Delta^{\prime}} \omega= \pm r \pi$, as we observed earlier that the symplectic area only depends of the summits of the triangle. There is a converse statement.

THEOREM 4.7. Let $\sigma_{1}, \sigma_{2}, \sigma_{3} \in \partial \mathcal{D}$ be three summits of an ideal triangle $\Delta$ such that $\int_{\Delta} \omega=r \pi$. Then there exists a unique tight holomorphic totally geodesic imbedding $\rho: \widetilde{\mathcal{D}} \rightarrow \mathcal{D}$ that

$$
\sigma_{1}=\rho(+1), \quad \sigma_{2}=\rho(-1), \quad \sigma_{3}=\rho(-i) .
$$

The image $\rho(\widetilde{\mathcal{D}})$ is the set of fixed points in $\mathcal{D}$ of the stabilizer in $\operatorname{Hol}(\mathcal{D})$ of the three summits.

The proof will be divided in several steps, with somme intermediate lemmas needed for the proof.

Step 1. Reduction to tube-type domains.

Clearly, by Theorem $4.2, \Delta$ must be of type $r$, so that the summits of $\Delta$ are points of the Shilov boundary $S$. Using the action of $G$, we may assume that the endpoints of one side of $\Delta$ are $E^{r}$ and $-E^{r}$. The third summit, say $\sigma_{3}$ is a point of the Shilov boundary $S$. We make free use of notation in section 1 . Recall in particular that $P$ denotes the projection of $\mathfrak{p}_{+}$on $\mathfrak{p}_{+}^{T}$. By Proposition (1.3), $P \sigma_{3}$ belongs to $\overline{\mathcal{D}^{T}}$. The 
points $\left\{E^{r},-E^{r}, P\left(\sigma_{3}\right)\right\}$ are mutually tranverse in $\mathfrak{p}_{+}^{T}$, as can be deduced from (1.5) and so, although they are not necessarily the summits of some ideal triangle of $\mathcal{D}^{T}$, the "triple ratio" $\varphi^{T}\left(-E^{r}, E^{r}, P \sigma_{3}\right)$ is well-defined and equal to $\varphi\left(E^{r},-E^{r}, \sigma_{3}\right)=r \pi$, as a consequence of formula (1.5), formula (2.2) and its extension to ideal triangles. Hence we are reduced to a tube-type situation, for triples of the form $(e,-e, z)$, where $e$ is the unit of the Jordan algebra and $z \in \overline{\mathcal{D}}, z$ transverse to both $e$ and $-e$.

Step 2. Extremal values of the triple ratio in tube-type domains.

As we did in [C- $\varnothing]$, the easiest formulation of the results in the tube-type case is by use of the Jordan algebra formalism. So let $U$ be a simple Euclidean Jordan algebra. The associated bounded domain $\mathcal{D}$ can be described as

$$
\mathcal{D}=\left\{z \in U_{\mathbb{C}}|| z \mid<1\right\}
$$

where || stands for the spectral norm on $U_{\mathbb{C}}$. The Bergman kernel in this context is (up to a positive constant which we ignore) given by 1

$$
k_{\mathcal{D}}(z, w)=\operatorname{Det}(\operatorname{Id}-2 z \square \bar{w}+P(z) P(\bar{w}))^{-1} .
$$

Specialize this formula to the case where $w=t e$, where $t \in]-1,+1[$, and $z \in \mathcal{D}$. Then

$$
K(z, t e)=\operatorname{Det}\left(\operatorname{Id}-2 t L(z)+t^{2} P(z)\right)^{-1}=\operatorname{Det}(P(e-t z))^{-1} .
$$

For the normalized Bergman kernel, we have an even simpler formula. In this case $p=\frac{2 n}{r}$ is an integer, and $\operatorname{Det} P(x)=(\operatorname{det} x)^{\frac{2 n}{r}}$ (see $\left.[\mathrm{F}-\mathrm{K}]\right)$, and hence

$$
k(z, t e)=\operatorname{det}(e-t z)^{-1}
$$

LEMMA 4.8. Let $z \in \overline{\mathcal{D}}$ be transverse to both $e$ and $-e$. The triple ratio for the triangle $\{-e, e, z\}$ is well-defined and has value

$$
2 \arg \operatorname{det}(e+z)(e-z)^{-1}
$$

Proof. As $z$ is transverse to $e$, the expression $(e+z)(e-z)^{-1}$ is well-defined, and (up to a missing factor $i$ ) is nothing but the Cayley transform of $z$. Moreover the known properties of the Cayley transform (see $[\mathrm{F}-\mathrm{K}]$ ) show that $(e+z)(e-z)^{-1}$ belongs to

$$
R=R_{\Omega}=\Omega+i U \subset U_{\mathbb{C}}
$$

which is of course $\frac{1}{i} T_{\Omega}$, but the use of the right "half-plane" will make easier the formulation of the results to be explained now. Moreover, the point $-e$ is mapped to 0 . As the transversality condition for two points $z, w$ in $\bar{R}$ means $\operatorname{det}(z+\bar{w}) \neq 0$, the point $(e+z)(e-z)^{-1}$ belongs to

$$
\mathcal{R}=\bar{R} \cap U_{\mathbb{C}}^{\times}
$$

\footnotetext{
${ }^{1}$ The "P" used in this formula is the traditional notation for the quadratic map associated to the Jordan algebra structure, not to be confused with the projection of $\mathfrak{p}_{+}$on $\mathfrak{p}_{+}^{T}$.
} 
the set of invertible elements in the closure of $R$. Now if $x \in \Omega$ and $y \in U$, then $x+i y$ is invertible, so that $R \subset \mathcal{R}$, and one can easily see that $\mathcal{R}$ with the induced topology from $U_{\mathbb{C}}$ is simply connected. The function $\operatorname{det} z$ doesn't vanish on $\mathcal{R}$, so that one can define a continous determination of the argument of this function, and it is unique is we demand that $\arg \operatorname{det} x=0$ for $x \in \Omega$. We denote by $\arg$ det this determination. If $g \in G(\Omega)_{0}$ and $z \in U_{\mathbb{C}}$, then

$$
\operatorname{det}(g . z)=\chi(g) \operatorname{det} z
$$

where $\chi$ is a real character of $G(\Omega)_{0}$. It implies that $\chi(g)>0$, and so the function arg det is invariant by $G(\Omega)_{0}$.

Lemma 4.9. Let $z=x+i y \in \mathcal{R}$. Then

$$
-r \frac{\pi}{2} \leq \arg \operatorname{det}(z) \leq r \frac{\pi}{2}
$$

Proof. Assume first that $z=x+i y$ belongs to the open right half-space $R$. As $x \in \Omega$, we may write

$$
x+i y=P\left(x^{\frac{1}{2}}\right)\left(e+i P\left(x^{-\frac{1}{2}}\right) y\right)
$$

so that $\arg \operatorname{det}(x+i y)=\arg \operatorname{det}\left(e+i y^{\prime}\right)$ with $y^{\prime} \in U$. Now by the spectral theorem in $U$, there is a Jordan frame $\left\{c_{j}\right\}_{1 \leq j \leq r}$ and real numbers $\lambda_{j}$ such that $y^{\prime}=\sum_{j=1}^{r} \lambda_{j} c_{j}$. But then for any $t, 0 \leq t \leq 1$,

$$
\operatorname{det}\left(e+i t y^{\prime}\right)=\prod_{j=1}^{r}\left(1+i t \lambda_{j}\right)
$$

so that

$$
\arg \operatorname{det}\left(e+i y^{\prime}\right)=\sum_{j=1}^{r} \arg \left(1+i \lambda_{j}\right)
$$

from which the (strict) inequality follows easily for all $z \in R$. The lemma follows by continuity.

LEMMA 4.10. Let $x+i y \in \mathcal{R}$, and assume that $\arg \operatorname{det}(x+i y)=r \frac{\pi}{2}$. Then $x=0$ and $y \in \Omega$.

Proof. First let $\left(y_{t}\right)_{t \in \mathbb{R}}$ be a family of elements of $U$ and assume that

$$
\arg \operatorname{det}\left(e+i y_{t}\right) \longrightarrow r \frac{\pi}{2}
$$

as $t \rightarrow+\infty$. Let $\mu_{j}(t), 1 \leq j \leq r$ be the eigenvalues (repeated with multiplicities) of $y_{t}$. Then $\arg \operatorname{det}\left(e+i y_{t}\right)=\sum_{j=1}^{r} \arg \left(1+i \mu_{j}(t)\right)$. So the limit condition forces $\mu_{j}(t) \rightarrow+\infty$ for every $j, 1 \leq j \leq r$, hence $\mu_{j}(t)>0$ for $t$ large enough. Hence $y_{t} \in \Omega$ for $t$ large enough.

Now let $z=x+i y \in \mathcal{R}$. Let $t>0$. As $x \in \bar{\Omega}$, the element $x+\frac{1}{t} e$ is in $\Omega$, and so we can write

$$
x+\frac{1}{t} e+i y=P\left(\left(x+\frac{1}{t} e\right)^{\frac{1}{2}}\right)\left(e+i P\left(\left(x+\frac{1}{t} e\right)^{-\frac{1}{2}}\right) y\right)
$$


Set $y_{t}=P\left(\left(x+\frac{1}{t} e\right)^{-\frac{1}{2}}\right) y$. Then

$$
\arg \operatorname{det}\left(e+i y_{t}\right)=\arg \operatorname{det}\left(x+\frac{1}{t} e+i y\right) \longrightarrow r \frac{\pi}{2}
$$

as $t \rightarrow \infty$. From the result just obtained, this forces $y_{t} \in \Omega$ for $t$ large enough, but this clearly implies $y \in \Omega$. But now, this implies that

$$
\arg \operatorname{det}\left(P\left(y^{-\frac{1}{2}}\right) x+i e\right)=r \frac{\pi}{2}
$$

There is a Jordan frame $\left(c_{j}\right)_{1 \leq j \leq r}$ such that $P\left(y^{-\frac{1}{2}}\right) x=\sum_{j=1}^{r} \lambda_{j} c_{j}$, with $\lambda_{j} \geq 0$. Then

$$
\arg \operatorname{det}\left(P\left(y^{-\frac{1}{2}}\right) x+i e\right)=\arg \operatorname{det}\left(\sum_{j=1}^{r}(\lambda+i) c_{j}\right)=\sum_{j=1}^{r} \arg \left(\lambda_{j}+i\right)
$$

and this equals $r \frac{\pi}{2}$ only if $\lambda_{j}=0$ for every $j, 1 \leq j \leq r$, so that $P\left(y^{-\frac{1}{2}}\right) x=0$, and hence $x=0$.

Step 3. Proof of the existence of a tight imbedding.

Lemma 4.10 is the key to the proof of the existence of $\rho$. In fact, set $z=P \sigma_{3}$. The triple ratio for $\{e,-e, z\}$ is $r \pi$. Hence, Lemma 4.8. and Lemma 4.10 imply, after inverse Cayley tranform, that the point $z$ has to be in $S^{T}$, the Shilov boundary of $\mathcal{D}^{T}$ and the triple ratio for $\{e,-e, z\}$ is nothing but ( $\pi$ times) the Maslov index of $\{e,-e, z\}$ in $S^{T}$ as defined in [C- $\left.\varnothing\right]$. This Maslov index has value $r$ only in the case where the triple $\{e,-e, z\}$ is conjugate to the triple $\{e,-e,-i e\}$. For this particular triple, the embedding of the unit disc $\widetilde{\mathcal{D}}$ in $\mathcal{D}^{T}$ given by $w \longmapsto w e$ is clearly tight and satisfies the desired relations. By conjugacy, this is also true for the triple $\{e,-e, z\}$. Now, as $z=P \sigma_{3}$ belongs to $S^{T}$, Proposition 1.5 implies that $P \sigma_{3}=\sigma_{3}$, and this shows that the summits of the ideal triangle we started with are the image of $1,-1,-i \in \widetilde{\mathcal{D}}$ under a tight imbedding of $\widetilde{\mathcal{D}}$ in $\mathcal{D}$.

Step 4. Some lemmas for the Siegel domain realization.

To prove the uniqueness statement, it clearly suffices to characterize the image of the tight embedding as the set of fixed points of the stabilizer of the three summits. It is easier to use the realization of the domain as a Siegel domain of type II. We first set notation (following more or less the setting of section 4) and prove a few lemmas.

So, let $U$ be a Euclidean Jordan algebra, with positive cone $\Omega$, and let $V$ be a complex vector space. Let $H: V \times V \longrightarrow U^{\mathbb{C}}$ be a Hermitian form which is $\Omega$-positive in the sense that $H(v, v) \in \bar{\Omega} \backslash\{0\}$ for all $v \in V, v \neq 0$. Consider the associated Siegel domain of type II

$$
\mathcal{S}=\left\{(u, v) \in U^{\mathbb{C}} \times V \mid \Im u-H(v, v) \in \Omega\right\}
$$

The group $\operatorname{Aff}(\mathcal{S})$ of holomorphic affine transformations of $\mathcal{S}$ consists of all transformations of the form

$$
\left\{\begin{array}{l}
u \longmapsto g u+2 i H(b, l v)+i H(b, b)+a \\
v \longmapsto l v+b
\end{array}\right.
$$


where $a \in U, b \in V, g \in G(\Omega), l \in G L(V)$, and $g, l$ satisfy the relation

$$
g H\left(v, v^{\prime}\right)=H\left(l v, l v^{\prime}\right) \quad \forall v, v^{\prime} \in V .
$$

Let

$$
G_{0}=\left\{(g, l) \in G(\Omega) \times G L(V) \mid g H\left(v, v^{\prime}\right)=H\left(l v, l v^{\prime}\right), \forall v, v^{\prime} \in V\right\}
$$

and let $\mathfrak{g}_{0}$ be the Lie algebra of the group $G_{0}$.

Now we take into account that $\mathcal{S}$ is a symmetric domain (for explicit necessary and sufficient conditions for such a Siegel domain of type II see [S] ch. V), which moreover is assumed to be irreducible. Let $\operatorname{Hol}(\mathcal{S})$ be the group of all holomorphic diffeomorphisms of $\mathcal{S}$. The group $\operatorname{Aff}(\mathcal{S})$ is a maximal parabolic subgroup of $\operatorname{Hol}(\mathcal{S})$, and $G_{0}$ is its Levi component.

LEMMA 4.11. The representation of $\mathfrak{g}_{0}$ on $U$ (resp. $V$ ) are irreducible as real representations.

For a proof, see [S] ch III, Prop. 4.4.

The space $V$ has a natural inner product defined by

$$
h(v, v)=<H(v, v), e>
$$

where $<,>$ is the standard Eulidean inner product on $U$. To these data is associated a selfadjoint representation $\Phi$ of the Euclidean Jordan algebra on $V$, defined by the formula

$$
\forall v \in V, \quad h(v, \Phi(u) v)=<H(v, v), u>.
$$

For each $u \in U$, the map $\Phi(u)$ is self-adjoint and satisfies

$$
\Phi\left(u u^{\prime}\right)=\frac{1}{2}\left(\Phi(u) \Phi\left(u^{\prime}\right)+\Phi\left(u^{\prime}\right) \Phi(u)\right)
$$

Notice also the following formula

$$
H\left(\Phi(u) v, \phi(u) v^{\prime}\right)=P(u)\left(H\left(v, v^{\prime}\right)\right)
$$

for all $u \in U, v, v^{\prime} \in V$. For $u \in U^{\times}, P(u)$ belongs to the group $G(\Omega)$ (see [F-K] Prop. III.2.2) and hence the couple $(P(u), \Phi(u))$ belongs to $G_{0}$.

LEMma 4.12. Let $l \in G L(V, \mathbb{C})$. The couple (Id, $l)$ belongs to $\operatorname{Aff}(\mathcal{S})$ if and only if $l \in \mathbb{U}(V, h)$ and $l$ commutes to the representation $\Phi$.

If $(\operatorname{Id}, l)$ is an element of $\operatorname{Aff}(\mathcal{S})$, then for all $v, v^{\prime} \in V$ we have

$$
H\left(v, v^{\prime}\right)=H\left(l v, l v^{\prime}\right)
$$

By taking the inner product with the neutral element $e$ of $U$, this gives the fact that $l$ is unitary. Now for $u \in U$, we have

$$
H\left(\Phi(u) v, \Phi(u) v^{\prime}\right)=P(u)\left(H\left(v, v^{\prime}\right)\right)=P(u)\left(H\left(l v, l v^{\prime}\right)\right)=H\left(\Phi(u) l v, \Phi(u) l v^{\prime}\right)
$$

Hence, by taking the inner product with the unit $e$

$$
h\left(\Phi\left(u^{2}\right) v, v^{\prime}\right)=h\left(\Phi(u) v, \Phi(u) v^{\prime}\right)=h\left(\Phi(u) l v, \Phi(u) l v^{\prime}\right)=h\left(l^{-1} \Phi\left(u^{2}\right) l v, v^{\prime}\right)
$$


which shows that $l$ commmutes to $\Phi\left(u^{2}\right)$. As $u$ was arbitrary and as the set of squares generate $U$, the element $l$ does commute to the representation $\Phi$. The proof of the converse statement is similar.

LEMma 4.13. The group generated by $\left\{(P(x), \Phi(x)), x \in U^{\times}\right\}$and $\{(\mathrm{Id}, l)|l \in \mathbb{U}(V, h)| l$ commutes to $\Phi\}$ is an open subgroup of $G_{0}$.

The group generated by $\left\{P(x) \mid x \in U^{\times}\right\}$is an open subgroup of $G(\Omega)$ (see [Sp], where it is called the inner structure group of $U$ and shown to be the neutral component for the Zariski topology of the structure group of $U$ ). The statement now follows from this remark and Lemma 4.12.

Step 5. Determination of the fixed points of the stabilizer of the three summits

As a consequence of what we proved in step 3, we may assume, after a Cayley transform, that the three summits of the triangle are "the point at infinity", the point $(0,0)$ and the point $(e, 0)$. The stabilizer of the the point at infinity in the group $\operatorname{Hol}(\mathcal{S})$ is the group $\operatorname{Aff}(\mathcal{S})$. The stabilizer in $\operatorname{Aff}(\mathcal{S})$ of $(0,0)$ is the subgroup $G_{0}$, and the stabilizer of $(e, 0)$ in $G_{0}$ is easy to determine. It is the subgroup

$$
C=\left\{(k, l) \in \operatorname{Aut}(U) \times \mathbb{U}(V, h) \mid k H\left(v, v^{\prime}\right)=H\left(l v, l v^{\prime}\right), \quad \forall v, v^{\prime} \in V\right\} .
$$

which acts linearly in $U \times V$.

LEMMA 4.14. The fixed points of $C$ in $U^{\mathbb{C}} \times V$ are

$$
\{(\zeta e, 0) \mid \zeta \in \mathbb{C}\}
$$

Obviously, the points of the form $(\zeta e, 0)$ are fixed under $C$. Conversely, assume that $(u, v) \in U \mathbb{C} \times V$ is a fixed point under the action of $C$. For $x \in U$ satisfying $x^{2}=e$, the element $(P(x), \Phi(x))$ is in $C$. Hence for $t=\Re u$ or $t=\Im u$, we have $P(x) t=t$ for all $x \in U$ such that $x^{2}=e$. Now assume $t$ is not a multiple of the unit $e$. This means that there exists a Jordan frame $\left\{c_{i}\right\}_{1 \leq i \leq r}$ such that $t=\sum_{i=1}^{r} t_{j} c_{j}$, with at least one pair $(j, k)$ with $j \neq k$ such that $t_{j} \neq t_{k}$. By [F-K], prop. IV.1.4, there exists an element $w_{j, k} \in V\left(c_{j}+c_{k}, 1\right)$ such that $w_{j, k}^{2}=c_{j}+c_{k}$ and $P\left(w_{j, k}\right) c_{j}=c_{k}, P\left(w_{j, k}\right) c_{k}=c_{j}$. Now let

$$
\widetilde{w}_{j, k}=\sum_{i \neq j, k} c_{i}+w_{j, k}
$$

Then

$$
\widetilde{w}_{j, k}^{2}=e, P\left(\widetilde{w}_{j, k}\right) c_{i}=c_{i} \text { for } i \neq j, k, P\left(\widetilde{w}_{j, k}\right)\left(c_{j}\right)=c_{k}, P\left(\widetilde{w}_{j, k}\right)\left(c_{k}\right)=c_{j}
$$

and hence $P\left(\widetilde{w}_{j, k}\right) t \neq t$. This forces $t$ to be a multiple of $e$, and hence there exists $\zeta \in \mathbb{C}$ such that $u=\zeta e$. Now for $v$, we get

$$
\forall x \in U \mid x^{2}=e, \quad \Phi(x) v=v .
$$

Now any idempotent element $c$ in $U$, one has $(e-2 c)^{2}=(-e+2 c)^{2}=e$, so that $c$ can be written as $c=\frac{1}{4}(-e+2 c)-\frac{1}{4}(e-2 c)$, and hence $\Phi(c) v=v$ for any idempotent, so that $\Phi(x) v=v$ for any $x \in U$. Hence the space $W$ of fixed points of $C$ in $V$ is stable by all $\Phi(x), x \in U$. By Lemma 4.13 , it is stable by an open subgroup of $G_{0}$. By Lemma 4.11, it means that either $W=\{0\}$ or $W=V$. But the map $x \mapsto \Phi(x)$ is 
injective (as $U$ is simple), and hence $W=V$ forces $U=\mathbb{R}$. But then the commutant of $\Phi$ is all $\mathbb{U}(V)$ and so $W=V$ forces $W=V=\{0\}$. In any case, we get $W=\{0\}$. Hence $v=0$ and so the fixed points are all of the prescribed form.

So, the fixed points in $\mathcal{S}$ of the stabillizer $C$ of the three summits is the set

$$
\{(\zeta e, 0), \Im \zeta>0\}
$$

The last statement of Theorem 4.7 follows by inverse Cayley transform. So the image $\rho(\widetilde{\mathcal{D}})$ is well-determined. It implies the uniqueness of $\rho$ up to a right composition by an element of $\operatorname{Hol}(\widetilde{\mathcal{D}}) \simeq P S U(1,1)$. But the stabilizer in $P S U(1,1)$ of the three points $1,-1,-i$ reduces to the identity. The uniqueness statement follows.

5. The Gromov norm of the Kaehler class of a compact locally symmetric Hermitian manifold. Let us recall the definition of the Gromov norm of a singular cohomology class. Let $X$ be a topological space and $c$ a singular cochain on $X$. Then define

$$
\|c\|_{\infty}=\sup _{\Delta}\{|c(\Delta)|\}
$$

where $\Delta$ runs through all singular simplices in $X$ (the quantity may be $+\infty$ ). If $\alpha$ is a singular cohomology class on $X$, then define the Gromov norm of $\alpha$ to be

$$
\|\alpha\|_{\infty}=\inf _{c}\left\{\|c\|_{\infty}\right\}
$$

where $c$ runs through all singular cocycles representing $\alpha$ (it is in fact a pseudo-norm).

Let $X$ be a compact locally symmetric Hermitian manifold with $\mathcal{D}$ as universal covering. Then $X=\mathcal{D} / \Gamma$ where $\Gamma$ is a certain discrete, torsion-free, co-compact subgroup of $G$. The Kaehler form $\omega$ induces a Kaehler form $\omega_{X}$ on $X$, and hence a real cohomology class $[\omega]_{X} \in H^{2}(X, \mathbb{R})$.

Theorem 5.1. Let $X$ and $\omega$ as before. Then

$$
\left\|[\omega]_{X}\right\|_{\infty}=r \pi
$$

Corollary 5.2. Let $S$ be a Riemann surface of genus $g>1, X$ and $\omega$ as above, and $f: S \longrightarrow X$ be a continuous map. then

$$
\left|\int_{S} f^{*} \omega_{X}\right| \leq 4 r(g-1) \pi
$$

The proof of the theorem and of the Corollary is the same as in [D-T]. The corollary is an easy consequence of the inequality $\left\|[\omega]_{X}\right\|_{\infty} \leq r \pi$ and an estimate of the (dual) Gromov norm of the fundamental homology class of $S$ (see [Gr]). To estimate the norm, we can first use the technique of "straightification" and properties of the Gromov norm (see [Gr]). It follows that

$$
\left\|[\omega]_{X}\right\|_{\infty} \leq \sup _{\Delta}\left|\int_{\Delta} \omega\right|
$$


where $\Delta$ runs through all geodesic triangles in $\mathcal{D}$, so that the inequality follows from Theorem 3.1. To prove that $\|[\omega]\|_{\infty}=r \pi$, it is sufficient by the proportionality principle $([\mathrm{Gr}])$ to construct an example of a discrete torsion-free, co-compact subgroup of $G$ for which the equality can be proven. The construction of such a subgroup $\Gamma$ follows the construction given in [Bo]. At the same time, one constructs a holomorphic totally geodesic map $\rho$ from the unit disc into $\mathcal{D}$ which is tight in our terminology, and a discrete co-compact, torsion-free subgroup $\widetilde{\Gamma}$ of $P S U(1,1)$ such that $\rho$ gives rise to a map $\tilde{\rho}$ (holomorphic, totally geodesic) from $S=\widetilde{\mathcal{D}} / \widetilde{\Gamma}$ into $\mathcal{D} / \Gamma$. By computing $\int_{S} \widetilde{\rho}^{*} \omega$ one sees that the maximum is obtained in the corollary, which in turn implies that $\left\|[\omega]_{X}\right\|_{\infty}=r \pi$. Details are left to the reader.

\section{REFERENCES}

[B-I] BURger M. AND IozzI A., Bounded Kähler class rigidity of actions on Hermitian symmetric spaces, (2002) preprint.

[B-I-W] Burger M., IOZzI A. AND WiEnhaRd A., Surface group representations with maximal Toledo invariant, to appear.

[Bo] Borel A., Compact Clifford-Klein forms of symmetric spaces, Toplogy, 2 (1963), pp. 111-122.

[B-G-G] Bradlow S., Garcia-Prada P. and Gothen P., Surface group representations, Higgs bundles and holomorphic triples (2002) preprint.

[C-Ø] CleRC J-L. AND ØRSTed B., The Maslov index revisited, Transformation Groups, 6 (2001), pp. 303-320.

[D-T] Domic A. AND Toledo D., The Gromov norm of the Kaehler class of symmetric domains, Math. Ann., 276 (1987), pp. 425-432.

[F-K] Faraut J. And Korányi A., Analysis on symmetric cones, Oxford Mathematical Monographs, Clarendon Press, Oxford (1994).

[F-al] FAraut J. ET AL., Analysis and Geometry on Complex homogeneous domains, Progress in Mathematics, 185, Birkhäuser Verlag, Boston (2000).

[Gol] Goldman W., Complex hyperbolic geometry, Oxford Math. Monographs (1999) Clarendon Press, Oxford.

[Got] Gothen P., Components of spaces of representations and stable triples, Topology, 40 (2001), pp. 823-850.

[Gr] Gromov M., Volume and bounded cohomology, Publ. Math. IHES, 56 (1982), pp. 5-99.

[H] HERNÁNDEZ L., Maximal representations of surface groups in bounded symmetric domains, Trans. Amer. Math. Soc., 324 (1991), pp. 405-420.

[M] Monod N., Continuous bounded cohomology on locally compact groups Lecture Notes in Math., 1758 (2001), Springer Verlag.

[P] Piatetsky-Shapiro I.I., Géométrie des domaines classiques et théorie des fonctions automorphes, Dunod, Paris (1966).

[S] SATAKe I., Algebraic structures of symmetric domains, Kanô Memorial Lectures 4, Iwanami Shoten and Princeton University Press, Princeton (1980).

[Sp] Springer T.A., Jordan algebras and algebraic groups, Erg. Math. 75, Springer verlag, New-York (1973).

[T1] Toledo, D., Harmonic mapings of surfaces to certain Kaehler manifolds, Math. Scand., 45 (1979), pp. 13-26.

[T2] Toledo, D., Representations of surface groups in complex hyperbolic geometry, J. Diff. Geom., 29 (1989), pp. 125-133.

[W] Wells R.O., Differential Analysis on complex manifolds, Prentice-Hall (1973). 
